\title{
Routing Protocols in Vehicular Ad hoc Networks: Survey and Research Challenges
}

\author{
Kayhan Zrar Ghafoor and Marwan Aziz Mohammed \\ Faculty of Engineering, Koya University, University Park, Danielle Mitterrand \\ Boulevard, Koya KOY45, Kurdistan Region - F.R. Iraq \\ Tel: +964(0)7504499850Ｅ-mail: kayhan@ieee.org \\ Jaime Lloret \\ Dept. of Communications, Polytechnic University of Valencia \\ Camino Vera s/n, 46022, Valencia, Spain \\ Kamalrulnizam Abu Bakar
}

Faculty of Computer Science and Information Systems, Universiti Teknologi Malaysia, 81310 UTM Skudai, Johor D. T, Malaysia

Zaitul M. Zainuddin

Faculty of Science, Universiti Teknologi Malaysia, 81310 UTM Skudai, Johor D. T, Malaysia

Received: September 4, 2013 Accepted: October 25, 2013 Published: December 31, 2013

DOI: $10.5296 / n p a . v 5 i 3.4134$

URL: http://dx.doi.org/10.5296/ npa.v5i3.4134

\begin{abstract}
A Vehicular Ad hoc Network (VANET) is a type of wireless ad hoc network that facilitates ubiquitous connectivity between vehicles in the absence of fixed infrastructure. Multi-hop routing and beaconing approaches are two important research challenges in high mobility vehicular networks. Routing protocols are divided into two categories of topology-based and position-based routing protocols. In this article, we perform a comparative study among the existing routing solutions, which explores the main advantages and drawbacks behind their design. After implementing the representatives of geographical and topology routing protocols, we analyze the simulation results and discuss the strengths and weaknesses of these routing protocols in regard to their suitability to vehicular networks. Lastly, we discuss the open issues and research directions related to VANET routing protocols.
\end{abstract}

Keywords: VANET, Topology-based Routing, Position-based Routing, V2V Communications 


\section{Introduction}

Recently, the growth in the number of vehicles on the road has put great stress on transportation systems. This abrupt growth of vehicles has made driving unsafe and hazardous. Thus, existing transportation infrastructure requires improvements in traffic safety and efficiency. To accomplish this, Intelligent Transportation Systems (ITS) have been considered to enable such diverse traffic applications as traffic safety, cooperative traffic monitoring and control of traffic flow. These traffic applications would become realities through the emergence of VANET because it is considered as a network environment of ITS. The increasing necessity of this network is an impetus for leading car manufacturers, research communities and governments to increase their efforts toward creating a standardized platform for vehicular communications. In particular, the $5.9 \mathrm{GHz}$ spectrum band has been allocated for licensed Short Range Communication (DSRC) between vehicles. In addition, in the near future, more vehicles will be embedded with devices that facilitate communication between vehicles, such as Wireless Access in Vehicular Environment (WAVE) [1]. When vehicles are equipped with WAVE, they can communicate with nearby cars and access points within their coverage area. Since vehicles have limited short radio range, they cannot cover large scale areas unless they use multi-hop routing protocols, which are significant handing over from traffic safety applications that require short distance coverage- to wide area coverage.

In vehicular environments, current state of the art routing protocols designed for Mobile Ad hoc Networks (MANET), Ad hoc On Demand Distance Vector (AODV) [2]; Dynamic Source Routing (DSR) [3]; Optimized Link State Routing (OLSR) [4] are less preferable. These protocols are address based rather than position based i.e., discover and maintain the end-to-end path between source and destination. This leads to frequent break of the routes due to high mobility and uneven (sparse and dense) distribution of vehicles. As a result, the protocol suffers from control overhead, hence leads to low network performance.

The alternative routing scheme, which is suitable for vehicular environments, is geographical routing where vehicles' route data packets by considering the position of the destination $[5,6]$. This type of routing is more desirable in VANET for the following reasons. First, in the near future, vehicles will be embedded with Global Positioning System (GPS) and navigation systems, hence geographical routing achieves monumental success in VANET. Second, since geographical routing is stateless, it does not maintain established routes between the source and the destination; hence it is highly scalable and very robust against frequent topological changes.

Neighbor discovery is the crucial part of geographical rout-ing protocols. To achieve this, the geographical routing protocols assume that nodes broadcast periodic beacon messages to inform neighbor nodes about their address, location and other relevant information. In this proactive neighborhood awareness, each vehicle should maintain the 
up-to-date list of neighbor nodes. Otherwise, the outdated information problem of the neighbor list leads to miss the next candidate node or the node that has been chosen will move out the radio range.

Although there are few surveys (e.g, [7], [8], [9], [10], [11], [12]) on the state of arts routing protocols, a very recent survey is an imperative need to thoroughly shedding light on the emerging routing protocols. In [7], Li et al., discussed the unique challenges of routing over vehicular environments and surveyed the routing protocols and VANET mobility models. In [8], Bernsen et al. classify and critically compared the existing unicast routing protocols for VANETs. In [13], Willke et al. surveyed the inter-vehicular communication protocols as well as their characteristics are exposed. Along with that, they classified the inter vehicular communication applications in order to determine their relevance with inter vehicular communication protocols. However, these surveys lack of a comprehensive detail and simulation of the emerging routing protocols. Furthermore, discussions on vehicular network applications are not mature without complete coverage of recent routing protocols and their impact of the overall VANET architecture.

In this article, a concise description of the background of VANET routing protocols will be presented first, followed by an elaboration of the main types of topology-based and position-based routing protocols, and the differences between them. Then, the classification of each type of VANET's routing protocols will be demonstrated. That is, topology-based routing is divided into proactive and reactive routing protocols, whereas geographical routing is classified to delay tolerant, non-delay tolerant and hybrid routing protocols. After that, it provides a survey of recent state of the art routing protocols, the motivation behind their design and trace the influence of mobility model on these routing protocols. Furthermore, the performance of representatives of geographical and topology routing protocols are evaluated in the urban vehicular environment. Finally, the paper discusses the possible future research directions. The overall structures of routing protocols are depicted in Fig. 1.

\section{Previous Surveys of VANET Routing}

Packet routing is crucial for the design of VANET since several applications are relying on this unicast communication such as file sharing between two vehicles. Even though there are several articles on VANET routing, none of them extensively survey recently proposed routing protocols as well as thoroughly simulate current state of the arts. Ho. et al. [10] conducted a performance evaluation of representative of geographical and topology based routing protocols in urban vehicular scenario. While their survey performs comprehensive performance comparison of existing state of the arts, many of representative routing protocols are specifically designed for mobile ad hoc networks such as GPSR, AODV, LAR, DSR and GRID. In another attempt, the authors in [14] surveyed different VANET routing solutions that provide guarantee of Quality of Service. However, their survey consists of few and not up-to-date routing protocols. In a similar reviewing attempt, Liu Jiancai et al. in [15] 


\section{Macrothink}

reviewed traditional proactive and reactive routing protocols that were particularly designed for mobile ad hoc networks. Although their survey is more recent from that of [10], the reviewed material is not up-to-date and much different from that of [10]. Similar to [15], the work in [16] was reviewed address based routing protocols and they also experimented the effect of mobility models on routing protocols.

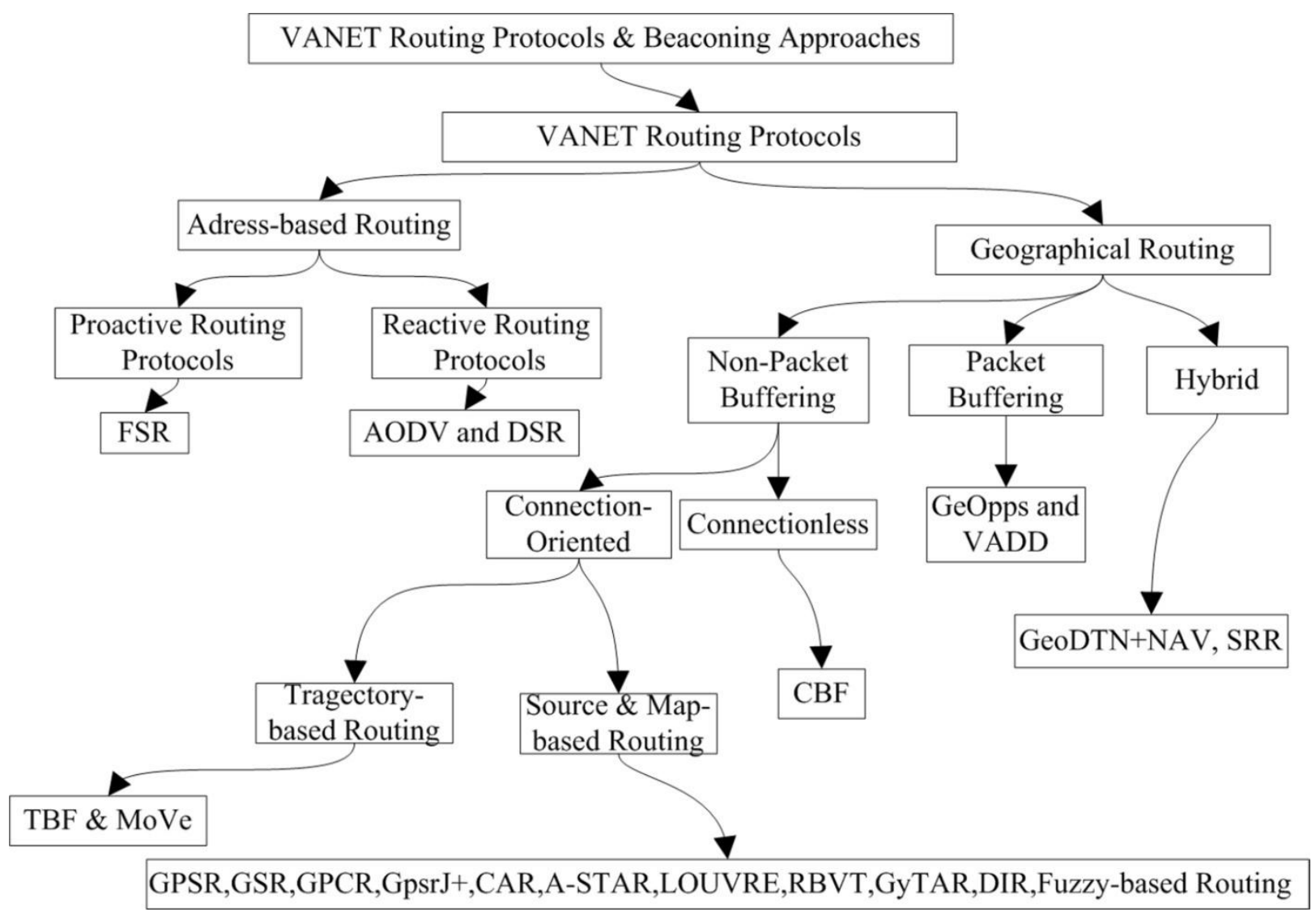

Fig. 1: Taxonomy of the literature on VANET routing protocols.

Following the trend, Jagadeesh et al. in [17] compared few of topology and position based routing solutions. Moreover, the authors did not only left some promising routing solutions, but they also did not provide simulation-based performance comparison between representatives of routing protocols. In [18], the authors were surveyed unicast, broadcast and multicast protocols that were specifically designed for VANET. They highlighted the key issues of routing protocols such as intermittent network connectivity and broadcast storm problem. Table I summarizes the existing survey works and shows the contribution of each article along with its timeline. 
TABLE I: Summary of existing survey on routing solutions in VANET

\begin{tabular}{|c|c|c|c|c|}
\hline Survey & Year of survey & Summary & Simulation & Realistic environment \\
\hline [9] & 2012 & $\begin{array}{l}\text { Surveyed and compared geographical } \\
\text { and topology based routing protocols }\end{array}$ & Simulation is not used & $\begin{array}{l}\text { Realistic propagation } \\
\text { model is not used }\end{array}$ \\
\hline$[10]$ & 2007 & $\begin{array}{l}\text { Surveyed representatives of } \\
\text { geographical and address based routing } \\
\text { protocols }\end{array}$ & $\begin{array}{l}\text { Their performance } \\
\text { comparison is based on } \\
\text { simulation }\end{array}$ & $\begin{array}{l}\text { The propagation model is } \\
\text { not realistic }\end{array}$ \\
\hline [7] & 2008 & $\begin{array}{l}\text { Surveyed the routing protocols and } \\
\text { VANET mobility models }\end{array}$ & Simulation is not used & $\begin{array}{l}\text { Realistic propagation } \\
\text { model is not used }\end{array}$ \\
\hline$[14]$ & 2008 & $\begin{array}{l}\text { Surveyed Quality of Service-aware } \\
\text { routing protocols }\end{array}$ & Simulation is not used & $\begin{array}{l}\text { Realistic propagation } \\
\text { model is not used }\end{array}$ \\
\hline$[15]$ & 2010 & $\begin{array}{l}\text { Surveyed proactive and reactive } \\
\text { routing protocols that were particularly } \\
\text { designed for mobile ad hoc networks. }\end{array}$ & Simulation is not used & $\begin{array}{l}\text { Realistic propagation } \\
\text { model is not used }\end{array}$ \\
\hline$[16]$ & 2008 & $\begin{array}{l}\text { Surveyed address based routing } \\
\text { protocols }\end{array}$ & Simulation is used & $\begin{array}{l}\text { Realistic propagation } \\
\text { model is not used }\end{array}$ \\
\hline [17] & 2011 & $\begin{array}{l}\text { Compared few of topology and } \\
\text { position based routing solutions }\end{array}$ & Simulation is not used & $\begin{array}{l}\text { Realistic propagation } \\
\text { model is not used }\end{array}$ \\
\hline$[18]$ & 2010 & $\begin{array}{l}\text { Surveyed unicast, broadcast and } \\
\text { multicast protocols }\end{array}$ & Simulation is not used & $\begin{array}{l}\text { Realistic propagation } \\
\text { model is not used }\end{array}$ \\
\hline
\end{tabular}

\section{VANET Routing Protocols}

The potential of VANET is immense, considering the monolithic number of vehicles on the roads today. This potency increases the necessity of vehicular networks among national government agencies, research communities and industry. As a result, this technology offers a bright prognosis and a future full of possibilities for transportation systems [19]. Moreover, VANET's multi-hop routing capability enables various applications and services that are odd for vehicular circumstances. They include, among others, safety-related applications to avoid accidents, comfort-related applications for vehicle-to-vehicle chatting, gaming and file sharing, and infotainment s. These emergent applications cannot be accomplished with the existing communication capability between vehicles. For the sake of efficient deployment of these applications, optimal and efficient routing protocols are required.

However, routing protocols is a key issue in vehicular networks despite their interesting applications. VANET routing protocols have been developed to forward data packets to specified destinations using en-route relay vehicles. Vehicular environments present odd communication characteristics on roadways, such as heterogeneous traffic distribution ranging from massive to a sparse number of vehicles, along with the high mobility of vehicles. Furthermore, even if a large number of vehicles exist, mobility constraints, by the roads and inter-sections, may lead to a spatial topology hole between platoons of vehicles. Due to these unique properties, developing routing protocols is a daunting task. On the other hand, a great deal of effort has been dedicated to the development of VANET-suited routing 
protocols by utilizing additional information sources which exist in vehicles (GPS, city maps and traffic density awareness). Position-based and topology-based routing protocols are elaborated in the following sections.

\section{Geographical and Topology-based Routing Protocols}

The main purpose of routing protocols is to facilitate communication between two nodes when they are out of radio communication range. In unicast routing protocols, route establishment consists of route construction, route optimization and route maintenance. In Fig. 1, recent state of the art unicast topology-based and position-based routing solutions are demonstrated. In particular, the topology-based routing protocols that have been proposed for MANET are adopted in VANET for comparison purposes. The next section focuses on the topology-based routing protocols.

\subsection{Topology-based Routing Protocols}

Topology-based routing protocols exploit the link state information that exists in the whole network to route data packets toward the destination. The issues surrounding these types of routing protocols have been researched widely over the past few decades. For instance, the MANET working groups of Internet Engineering Task Force (IETF) [20] have proposed routing solutions for such unpredictable mobile environments. Some of these investigations have been implemented in real environments, and they prove that these routing protocols perform well in static or moderate mobility of nodes. In general, topology-based routing protocols are classified into reactive and proactive solutions.

\subsubsection{Proactive Routing Protocols}

Proactive routing broad-casts periodic hello messages, like traditional routing in the internet, in order to determine the global view of the net-work topology, which is useful when route establishments are needed. However, established routes which are cached in each node might never be used. This leads to a waste of network bandwidth, especially in high node density. In addition, in proactive routing protocols, there is a trade-off between the freshness of cached routes and the frequency of message broadcasts. Frequent broadcast messages are useful in order for the packet carrier node to calculate efficient rout es to the specified destination. However, this is at the expense of high bandwidth consumption, which grants the channel for broadcast traffic. On the other hand, this type of routing is suitable for real time applications (delay sensitive services) since the route between a pair of sources and destinations is created beforehand. In other words, the source does not need to flood route discovery requests on demand as the route is established in the background. In spite of the low end-to-end latency of packet forwarding, the recovery of unused cached routes wastes massive bandwidth, especially in high mobile environments. 


\subsubsection{Fisheye State Routing (FSR)}

In [21] the authors developed an efficient link state algorithm that maintains the global knowledge of the network topology at each node, and disseminates the local information to the direct neighbor nodes instead of the whole network. In FSR protocol, the updates of link state information vary with the distance to-wards the destination. Fig. 2 shows the basic operation of FSR. That is, every node defines a boundary around itself. The inner boundary is formed by the closer nodes, and they receive the link state information with the highest frequency, whereas the further nodes broadcast the update with lower frequency. Thus, the FSR protocol exchanges the link state information frequently with the vicinity nodes, and with lower frequency for the further nodes. In this way, the nodes can get up-to-date link state information about the nearby neighbor nodes. Apparently, there is a trade-off between the reduction of overhead and the staleness of the link state information, leading to sub-optimal route selection.

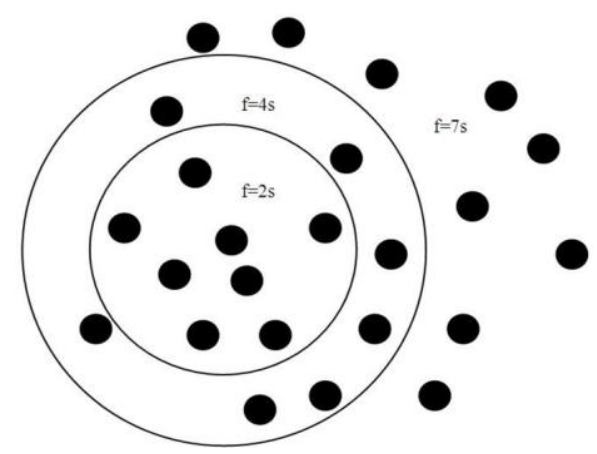

Fig. 2: This scenario shows different frequencies of link state updates for every boundaries.

\subsubsection{Destination Sequenced Distance-Vector (DSDV)}

In [22], the authors proposed the Destination Sequenced Distance-Vector which is based on the Bellman-Ford routing algorithm. Each mobile can communicate with the destination through its routing table that contains destination address and hop count to reach those destinations. Fields in the routing table save a sequence number by the destination. This number is helpful to avoid routing loop and staleness of the table. Flooding technique is utilized to update routing tables in each mobile node. Two types of routing update is used; full dump and incremental. Full dump floods the network with entire routing table whereas incremental method only floods those entries from routing table since last full dump update. In stable networks, incremental method is frequently used while full dump update method is dominant in fast moving network. In addition to the content of routing table, source node assigns a sequence number to the route update packet. The route with highest sequence number is used for data communication between a pair of source and destination. In case of equal sequence number of two routes, the shortest route will be chosen. 


\section{NI Macrothink}

\subsubsection{Optimized Link State Routing (OLSR)}

In [4], Clausen et al. proposed OLSR that uses Multipoint Relays (MPR) to optimize the link state by reducing the size of each forwarded packet and minimizing number of retransmissions in the network. Using MPR, each node selects one hope neighbors and considered MPR nodes. The non-MPR neighbor nodes process the received packets while the MPR nodes forward the received packets. The MPR set should be efficiently chosen in order to include minimum number of neighbors. This minimum set reduces number of broadcast packets within the network range.

\subsubsection{Reactive Routing Protocols}

The basic operation of proactive routing protocols works in reverse to the On-demand topology-based routing. This routing solution establishes a route when it is requested by a node to send packets to another node in the network. At this time, the node re-broadcasts the requested route establishment to find the intended destination. When the destination receives the query (or the en-route nodes know the path to it), it responds to the source for the purpose of route establishment between source and destination.

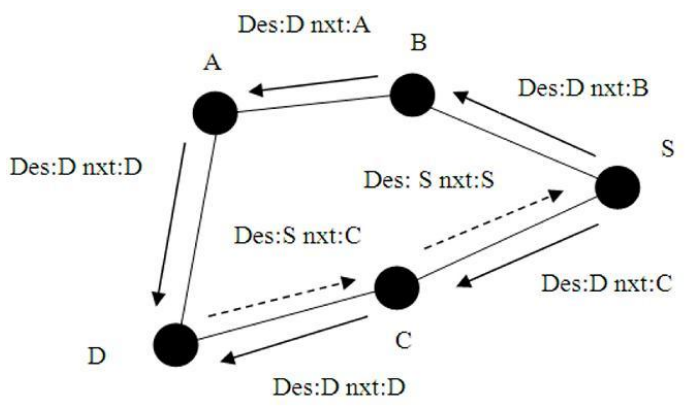

Fig. 3: AODV basic operation. The illustration of route establishment procedure.

\subsubsection{Ad Hoc On Demand Distance Vector Routing Protocol (AODV)}

In AODV, as demonstrated in Fig. 3, when a source node has data packets and intends to communicate with another node, it initiates the route discovery process in the network. As the source has no suitable route toward the destination, it broadcasts the Route Request (RREQ) message. This message is then forwarded by the en-route nodes until it reaches the destination node or the intermediate node which has a fresh route toward the destination. Upon receiving the RREQ, the destination responds to the source with a Route Reply (RREP) message. Furthermore, as the nodes receive the RREQ, they record the address of the RREQ sender in their routing table. This procedure is helpful as the destination learns the route to the source. In this way, the destination sent a RREP message through the complete recorded path, which is learned from the received RREQ message. The source maintains the established route as long as it is active.

For monitoring the route failure, the nodes predict the status of the link of its direct neighbors in the active routes. Every node can monitor the link status by utilizing the MAC 
layer handshaking mechanism or periodic HELLO messages. When this happens, the detecting node sends the route error message to the direct neighbor nodes which are present in its predecessor list. Then the inaccessible route notification is also sent to all nodes exist in the active route. In response to this, the source initiates another route discovery procedure to establish a new fresh route toward the destination.

As mentioning that, AODV maintains only the active route, thus it is less susceptible to bandwidth consumption compared with the proactive routing solutions. However, the main problem of AODV is the route discovery phase which introduces network overhead for each data packet. This problem becomes more crucial when the wireless link is unreliable or utilized as a bridge between two sub-networks such as in vehicular scenarios when a road connects two sites.

\subsubsection{Dynamic Source Routing Protocol (DSR)}

DSR is the source based routing protocol where the source records the sequence of intermediate nodes in a data packet which is transmitted toward the destination. The basic operation of the protocol consists of two phases, namely the route discovery and route maintenance processes. When a node needs to send data packets toward the destination, it first checks its cache for the existence of a route. If the route does not exist, it starts the route discovery phase by sending a query packet indicating the destination to its direct neighbor nodes. This packet records the ID (IP address) of the en-route nodes that have been traversed. In this way, the query packet carries the complete path followed by the packet. After the packet is received by the destination, it extracts the complete path to reply to the source. The destination may respond to the source with multiple route replies, and then keeps these routes as a backup in the event of route failure. In static or moderate mobility, in contrast to AODV, this characteristic makes DSR reliable for route failures. The main differences between AODV and DSR are as follows. First, in AODV, data packets record the destination address, but in DSR the data packets record the traversed route from source to destination. Thus, DSR performance suffers from routing overhead and leads to scalability issues in comparison to AODV. Second, the route reply packet records the address of the destination and the sequence number, whereas, in DSR the route reply packets carry the complete path that exists between the source and the destination. Fig. 4 illustrates the complete fundamental processes of DSR protocol.

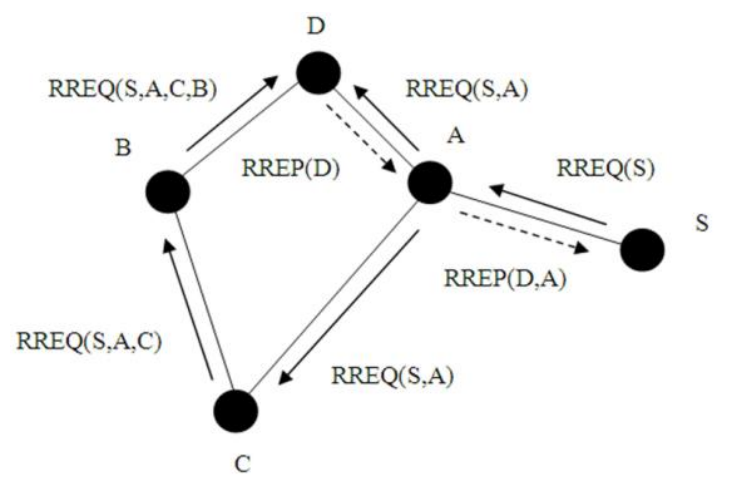

Fig. 4: DSR basic operation 


\subsubsection{Analysis and Evaluation of the Topology-based Routing Protocols}

The frequent use of message flooding into the network causes topology-based routing protocols to offer poor performance in VANET. This naive mechanism wastes a lot of bandwidth in the network. In particular, this effect is spectacular in dense vehicular scenarios where massive numbers of vehicles in the urban/highway want to exchange information. In other words, each vehicle shares local information with non-local nodes in the network. This type of naive flooding has several drawbacks. First, large amounts of channel bandwidth are consumed due to handshaking of the control messages, and little bandwidth remains for the applications, which may lead to broadcast storm problems [23]. Consequently, packet drop occurs in the network. Second, the established route may have a short life span due to high inter-channel variation with high mobility of vehicles.

In [24] the authors evaluated topology-based routing proto-cols in the urban vehicular scenario using packet level Network Simulator NS-2. They utilized the Manhattan topology to simulate the grid structure of the city map. The Intelligent-Driver Model (IDM) has been used to determine the speed of vehicles, adapted by the surrounded vehicles and the road topologies [25]. This simulation study shows that the AODV protocol performs the best compared to DSR and FSR, whereas FSR offers better performance compared with DSR. This is because the cached route in the source changes continuously due to high mobility. Furthermore, the performance of topology-based routing protocols degrades with increases in traffic density. This situation occurs in [24] as well as du e this issue other protocols having scalability issues.

Another direction of evaluation of these protocols in VANET is taken by [26], where they compared the GSR (which is explained in section), AODV and DSR in a realistic vehicular scenario (small part of a map of Berlin). In their evaluation, they consider the obstacle modeling in radio propagation model. Furthermore, they consider the space between streets as radio obstacles (e.g., buildings), where the radio signals cannot penetrate. An extensive performance evaluation shows that GSR is far superior compared with AODV and DSR. Moreover, the AODV again performed better compared to DSR due to massive bandwidth consumption, and high mobility causes frequent route breaks.

In the light of the aforementioned discussion regarding topology-based routing, it is clear that these types of protocols are less preferable in vehicular environments. In many vehicular scenarios, the life time of the established route between a source and a destination is very short, and setting up such a route may not be warranted. A much more sympathetic solution for VANET is geographical routing, where local in-formation only is enough for routing data packets, in a hop-by-hop basis, toward a destination. Indeed, it is conceivable that multi-hop routing can flourish with the support of geographical routing in vehicular networks.

\subsection{Geographical Routing Protocols}

Geographical routing primitively developed for packet radio networks in 1987 [27]. This type of routing received revived interest during the last few years in mobile, sensor, mesh and vehicular wireless networks [28, 29, 30, 31, 32]. In position-based (geographical) routing, 
packet forwarding decisions by a node are based mainly on the position of direct neighbors and the position of the packet's destination. These protocols, specifically developed for MANET, cannot be directly applied in vehicular networks. The principle is quite simple, because the movement of vehicles is constrained by the roads, and the paths are allowed by the environment (not free of movement like MANET nodes). Furthermore, unlike MANET, a large number of vehicles are travelling on the highway/urban scenarios. As a result, VANET routing protocols must utilize localized information to achieve the scalability requirements. Then, vehicles make packet forwarding decisions based solely on local information provided by nearby direct neighbors. This leads to less control overhead due to the suppression of the node's global knowledge of other parts of the network.

In geographical routing, knowing local information about direct neighbor nodes is counted as a preliminary requirement. Each vehicle obtains this information through periodic beacon messages based on one-hop broadcast. The beacon messages, which are exchanged between passing vehicles, include the speed, direction, position or other relevant in-formation. With such cognition, a vehicle can predict the connection time and link breakage accordingly. On the other hand, there are recent routing solutions based on reactive neighbor discovery, namely beaconless schemes, which are part of data packet forwarding.

Another issue of geographical routing in many services is the real time identification of the position of the mobile destination. This is because, unlike MANET, which relies on the IP address of the destination, most of the routing protocols in VANET forward packets to a specified position or area. Thus, the source needs to know the position of the destination for packet forwarding purposes. To do that, it is necessary to deploy a scalable location service to gain such position information. However, the design of location services is quite awkward due to scalability issues. Some geographical routing protocols utilize the query message which is included the position of the destination. Furthermore, CarTalk2000 [33] project proposed a location service based on different scopes where a vehicle broadcasts its location information to a limited number of hops. The updater frequency is inversely proportional with the distance, i.e., the further the distance between the source and other vehicles, the lower the frequency of updating. In addition, there are other distributed location services such as $[34,35,36]$ in which destinations update their location periodically. The source node will query the location servers to obtain the positional information of the destination. In this way, the source can forward data packets toward up-to-date destination positions.

In VANET, packet forwarding decisions are either based on the scores of the direct neighbor nodes or the pre-computed routes toward the destination. For the former, the routing protocol computes a suitable trajectory for the packets to follow. Since this approach considers a trajectory rather than individual next hops, it is very robust to the high mobility vehicular environments. Candidate nodes which are close to the pre-computed trajectory forward data packets toward the destination. However, in some cases, the created trajectory passes through sparse or disconnected vehicular networks. A more appealing solution to forward data packets in vehicular scenarios is to benefit from local information provided by direct neighbors. This type of packet routing is very common in geographical forwarding in which the data source utilizes neighborhood information. For instance, in greedy based 
routing, the source gives the higher score to the direct neighbor node which has the shortest distance to the destination. But, if there is no direct neighbor node of the current data source, then it simply drops the data packet.

VANET networks are characterized by heterogeneous traffic distributions, leading to disconnected vehicular networks. In this situation, the data packets eventually reach en-route vehicles that cannot forward data packets as planned. Some routing protocols assume that there are enough vehicles on the roads. In contrast to this routing solution, some protocols utilize the principle of Delay Tolerant Networks (DTN) [37], implying that if data packets are not delay sensitive, packets are carried and forwarded.

Other implicit characteristics of VANET are that nodes should follow road segments. They have to obey the traffic rules, the layout of the roads and the movement of the other vehicles in the area. Geographical routing protocols can exploit these features to predict future positions. Thus, they can make more robust packet forwarding decisions toward the destination. However, the staleness of the positional information leads to incorrect packet forwarding or future mobility predictions.

VANET geographical routing protocols can be classified based on a number of factors. Fig. 1, sub-classified geographical routing into three categories. Each category corresponds to the protocols and whether they use delay tolerant techniques or not. The first category (Packet Buffering) includes those routing protocols which consider intermittent connectivity, whereas the second category (Non-Packet Buffering) does not consider dis-connectivity of platoons of vehicles. Hybrid types of geographical routing protocols combine both of them to be suitable for uneven distribution of vehicles. In the following sections, these three sub-categories are explained:

\subsubsection{Packet Buffering based Geographical Routing Protocols}

Delay tolerant vehicular routing protocols, which are designed for VANET, are envisioned to be useful in many applications [38, 39, 40, 41, 42]. As mentioned earlier, vehicles are moving in constrained roads at high speed, and as a result, they suffer from frequent intermittent connectivity. This issue is addressed by designing vehicular DTN protocols; that is, when the data source has no contact with other direct neighbors, it temporarily stores the packet until it finds other opportunities to forward it (carry-and-forward strategy). Inside this group, the more notable routing protocols are Geographical Opportunistic Routing (GeOpps) [43] and Vehicle-Assisted Data Delivery (VADD) [41].

\subsubsection{Geographical Opportunistic Routing}

In [43], the authors proposed GeOpps, which is a geographical routing protocol which takes advantage of both vehicles' movement opportunity behavior and the vehicles' navigation system to give higher rank to vehicles that are moving closer to the final destination. It also utilizes the concept of carry-and-forward mechanism, so that when a vehicle reaches a disconnected network, it caches data packets until the suitable next hop is reached. 


\section{Ml Macrothink}

For packet forwarding decisions, packet carrier vehicles determine the closest point from the packet's destination. As demonstrated in Fig. 5, vehicle A computes the nearest points of routes $\mathrm{R} 1$ and $\mathrm{R} 2$ toward the destination $\mathrm{D}$. R1 has shorter distance to the destination, node $\mathrm{A}$ chooses R1 to forward data packets. Thus, given the nearest point and a map of the area, minimal time is required to forward packets toward the destination.

They also calculated the required time to deliver the packet by using a utility function. Once the vehicle is aware of its nearest point, it utilizes the map to compute the Estimated Time of Arrival (ETA) to the Nearest Point (NP). It then estimates the time required for a vehicle to travel from NP to the destination D.

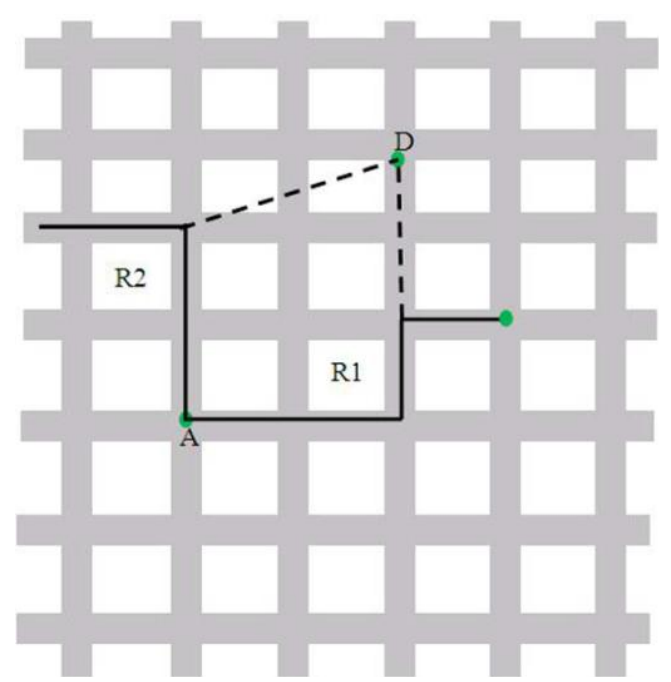

Fig. 5: Calculation of the nearest point from packet's destination for $R_{1}$ and $R_{2}$ paths.

The summation of these two estimated times gives Minimum Estimated Time for Delivery (METD) which is given by:

$$
M E T D=E T A \text { to } N P+\text { ETA from NP to D }
$$

GeOpp is designed to forward packets in delay tolerant networks, and its performance depends on the suggested route to the destination. Therefore, if the data source vehicle does not follow the route recommend by the navigation system, the packet forwarding decision might be sub-optimal. Since this protocol requires a navigation system for its packet forwarding, it is susceptible to privacy issues such as vehicle tracking. In addition, as witnessed by Cabrera et al. in [44], METD is not always suitable to be used as a criterion for packet forwarding towards the destination. More particularly, in heterogeneous traffic distribution scenarios although a specific trajectory has minimum estimated time, the packet gets stuck on that trajectory (in dense scenario) or a node might carry a packet and move away from the destination (in sparse scenario). To address this problem, the authors in [44] proposed an approach that gives priority to those nodes whose trajectory approaching the destination. The simulation results show the superiority of their proposed approach as compared to the GeOpps. 


\subsubsection{Vehicle-Assisted Data Delivery}

Zhao and Cao (2008) developed variations of VADD protocols. All of them employ the concept of carry-and-forward strategy and the predictable nature of vehicular mobility to improve routing protocols in VANET. More specifically, the main goal of VADD is to select the route with the smallest packet delay. The protocol's packet forwarding mechanism changes with the position of the packet carrier node. That is, a vehicle makes a decision at the intersection and forwards the packet to the road which has minimum packet delivery delay.

The estimation of packet delivery delay through certain roads is modeled and expressed by parameters such as road length, average vehicle velocity and road traffic density. M ore-over, in expectation of delivery time delays to the destination, the authors also utilized the delay estimation model of the next possible roads. Fig. 6 shows the concept of delivery delay estimation in VADD. A vehicle at intersection $\mathrm{n}$ calculates the road delay between intersections $\mathrm{n}$ and $\mathrm{m}(\mathrm{DNM})$, then computes the road delay between intersections $\mathrm{m}$ and $\mathrm{x}$ (DMX). In this way, each vehicle at an intersection can estimate the packet delivery delay of the oncoming roads.

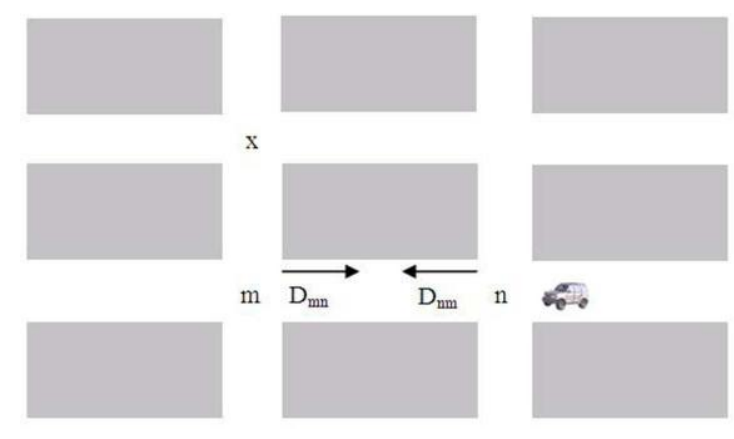

Fig. 6: VADD delay estimation model

The minimum delay estimation of different roads is solved by a set of linear system equations $(n \times n)$ using Gaussian elimination method, where $n$ is the number of junctions. Computational overhead is minimized by introducing a scope around the current position, reducing the number of junctions inside that area, and hence reducing the number of linear equations.

Once the junction node selects the next road, a packet carrier node at the road tries to find the next relay node. On straight-aways, the priority is given to a node which is closest to the next intersection, and the next junction is the one in the direction of the current packet carrier vehicle. In cases where there are no direct neighbor nodes within the transmission range of the packet carrier node, it caches the packet for another forwarding opportunity. Furthermore, after the performance evaluation, the VADD performs better compared with GPSR.

One of the main drawbacks of VADD is that its complexity grows with increasing numbers of junctions in the scoped area. The vehicles' decision within the scoped area is a trade-off between the computational complexity of solving a set of linear equations and the accuracy of estimated delay. 


\section{Macrothink}

\subsubsection{Non-Packet Buffering based Geographical Routing Protocols}

These types of routing protocols assume that there are always enough vehicles in the urban/highway vehicular scenarios.

Based on this assumption, packet carrier nodes do not reach disconnected networks. This group of routing is divided into two sub-categories: connectionless routing protocols and connection-oriented routing protocols.

\subsubsection{Connectionless Geographical Routing Protocols}

Connectionless routing solutions introduce protocols which they do not need to maintain the communication connections and the neighborhood information [45, 46, 47]. A notable geo-graphical routing protocol for this group is Contention-Based Forwarding (CBF) [48] and Guaranteed Delivery Beaconless Forwarding Scheme (GDBF).

\subsection{Contention Based Forwarding}

$\mathrm{CBF}$ is a greedy based geographical routing protocol that does not need periodic beacon transmissions. The packet carrier node, which runs CBF, does not score the direct neighbor nodes. Rather, it broadcasts the control frames to them, and they should decide individually whether to forward a packet or not. That is, the packet carrier node broadcasts the RTS frame containing it's and the destination position. Then the next relay node is selected by distributed timer-based next hop self election in the contention period. The winner (shortest reply time) of the contention phase is the node which has more geographical progress toward the destination. The contention winner broadcasts the CTS frame to the node, which is an originator of the RTS frame. At this time, the candidates nodes that hear this CTS frame cancel their timers and exit from the contention process. Fig. 7 depicts the concept of CBF, where vehicle N1 replies to the source first and becomes a next relay node because it has made more progress toward the destination.

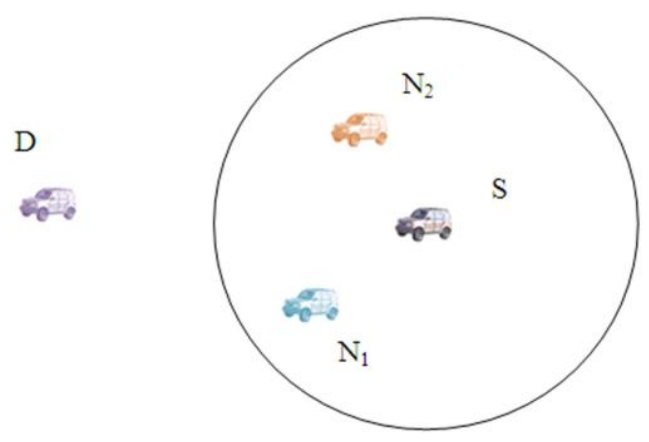

Fig. 7: CBF data packet forwarding. 
The proposed beaconless CBF is compared with GPSR with different beaconing generation interval in highway traffic scenario. When the frequency of beacon generation is $250 \mathrm{~ms}$, CBF delivers more packets than GPSR. As the beacon interval increases to $2000 \mathrm{~ms}$, CBF again performs better compared to GPSR. However, CBF has two drawbacks. First, they consider unit disc transmission range, which is unreliable in vehicular scenarios since the inter-channel between vehicles is susceptible to fading and shadowing effects. Second, they do not consider the unreliability and instability issues of packet forwarding by considering other parameters like power signal or direction of movement. Thus, this protocol may lead to sub-optimal results if the erasure channel is considered.

\subsection{Guaranteed Delivery Beaconless Forwarding Scheme (GDBF)}

The authors in [49] proposed a geographical forwarding scheme called Guaranteed Delivery Beaconless Forwarding Scheme (GDBF). In the proposed scheme, the relay node is selected through the use of control RTS/CTS frames of the MAC layer and waiting time function. In greedy mode, the candidate node which is closest to the destination responds to the source first.

When a source node has shortest distance to the destination as compared to the distance of direct neighbor nodes, the contention winner might be the node which is closer to the source. Thus, other nodes which overhear the CTS frame exit from the contention phase because there is a link established with the source. The GDBF could guarantee packet delivery as compared with the existing beaconless routing protocols.

Furthermore, the existing beaconless approaches either retransmit the whole data packet immediately, which might lead to the redundant retransmissions, or have duplicate packets.

In their analysis, they confirmed low routing overhead and high guaranteed delivery. However, they assumed ideal MAC layer and unit disc radio propagation in their performance evaluation. Since the wireless channels between vehicles in the urban environment are error prone due to high inter-channel variation, shadowing and fading effects, the aforementioned authors did not consider the quality of wireless channel and stability of packet forwarding. In addition, their proposed scheme is not designed for urban vehicular environments.

\subsubsection{Connection-Oriented Geographical Routing Protocols:}

In these routing solutions, a node needs to establish connection with single-hop neighbor nodes within its radio coverage. Thus, nodes in the same radio range maintain their local information by frequently sending beacon messages. This group is divided into trajectory-based routing solutions, and source and map based routing protocols.

\subsection{Trajectories-based Geographical Routing Protocols}

All vehicles are equipped with speedometers and odometers to measure their speed accurately. Furthermore, navigation systems which guide drivers to more suitable routes are 
very popular. Therefore, with this information, trajectory based geographical routing can forward data packets along the shortest path toward destination. In this type of routing, vehicles follow a pre-defined trajectory or imaginary curve to forward their data packets from the source to the destination. Common routing protocols belonging to this group are Trajectory-Based Forwarding (TBF) [50] and Motion Vector scheme (MoVe) [51].

\section{- Trajectory-Based Forwarding}

As mentioned earlier, the data source should forward data packets along a pre-computed trajectory. The trajectory is defined as some form of parametric equation. Usually this equation is included in the header of the message, which acts as a guide to route the message. The packet carrier node selects a direct neighbor which is the closest node to the specified trajectory. Evidently, to achieve more forwarding progress toward destination, the selected closest node to the trajectory must be in front of the current packet holder node. In low traffic density, the drawback of this solution is that it can not achieve local optimum; because it is possible packet holder nodes reach some points where there are no neighbor nodes.

\section{- Motion Vector Scheme}

MoVe is an opportunistic geographical routing protocol that forwards data packets with the help of velocity in-formation. This routing protocol assumes that all vehicles have knowledge about their own position, velocity vector and the destination. When a vehicle carries data packets to be forwarded, it broadcasts periodic beacon messages. As the direct neighbor nodes receive this broadcast traffic, they send a response message to the source. The response and beacon messages bear the senders' closest distance to the packet's destination. Finally, the packet is forwarded to a direct neighbor node which is predicted to have made more forward progress toward the destination. The main drawback of MoVe is that the velocity vector varies very quickly when there is high mobility of vehicles. Thus, the local minimum might occur.

\subsection{Source and Map based Routing}

In this section, the recent routing protocols used in VANET are briefly described, notably geographic routing and fuzzy logic-based routing protocols.

\section{- Greedy Perimeter Stateless Routing}

GPSR [52] is considered as a position-based routing because it utilizes the positions of the vehicles and the location of the packet's destination when making forwarding decisions. In addition, the GPSR protocol is known as stateless, because the intermediate vehicle employs the beacon message to collect the positions of their neighboring vehicles rather than using routing metrics. GPSR forwards packets in two modes: greedy mode and perimeter mode.

In greedy mode, an intermediate node receives a packet, and then selects a neighbor node that is geographically closest to the destination node. If an intermediate node has no other 


\section{Macrothink

neighbors closer to the destination than itself, it enters a local maximum. In this case, the packet will switch to the perimeter mode to recover from the local optimum. Fig. 8 shows an example of a local maximum. The source node $S$ has data packets, and is trying to determine neighbor nodes to forward its packet toward destination $\mathrm{D}$, but it can not find neighbor nodes ahead of itself. As a consequence, it fails to deliver data packets due to local maximum.

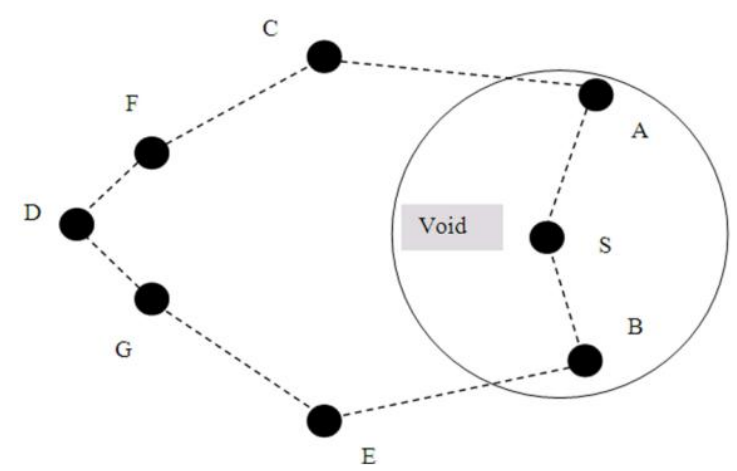

Fig. 8: Perimeter mode of GPSR protocol [53].

When the graph is planar, the GPSRs perimeter mode relays data packets by utilizing the right hand rule with respect to the starting vector constraint. The rule states that when a packet at intermediate node $\mathrm{x}$ switches to the perimeter mode, its next relay node $\mathrm{y}$ is the node that is sequentially counter clockwise to the virtual edge formed by $\mathrm{x}$ and the destination $\mathrm{D}$. Then, the next hop $\mathrm{z}$ is sequentially counter clockwise to the edge formed by $y$ and its previous node $x$. It is worth mentioning that if there are cross edges in the graph (the graph is not planar), the routing loop occurs. Thus, the performance of GPSR degrades when the graph is not planar [9].

In [54], the GPSR protocol is compared with DSR in highway vehicular scenario. In their simulation, DSR was counterproductive in terms of the packet delivery ratio when the communication range increases. This can be attributed to the fact that DSR maintains the route at the source, leading to bandwidth overhead in the network.

\section{- Geographic Source Routing (GSR)}

Lochert et al. (2003) in [26] proposed a geographical routing protocol named GSR. This routing mechanism integrates geographical routing supported by city maps. If the position of the destination, position of the source and the map of the city is given, GSR determines the number of junctions the packet should follow. Then the protocol applies Dijkstras algorithm to find the shortest possible path toward a destination. After determining a sequence of junctions, the protocol utilizes greedy routing to forward data packets at the roads. That is, a packet carrier node selects a candidate node which is closest to the next intersection. The protocol continues to forward packets in this way until the destination is reached or the life time of the packet is expired (Fig. 9). 


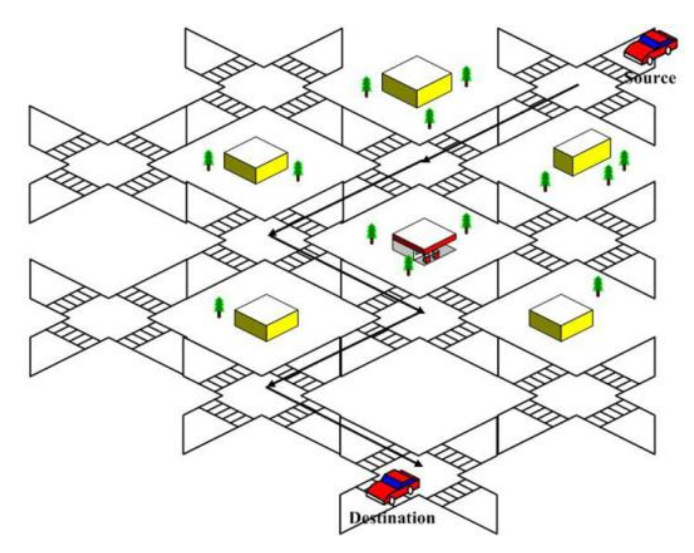

Fig. 9: Greedy Source Routing in urban environment.

Based on their simulation study, the GSR performs better than topology-based routing protocols like AODV and DSR in terms of end-to-end delay and successful delivery ratio. However, this routing mechanism neglects sparse vehicle scenarios in which there are not enough nodes for packet forwarding.

The drawbacks of GSR are that when the traffic density is sparse (connectivity between vehicles is low), its packet delivery ratio degrades. If a packet holding node cannot find any neighbor closer to the next junction, more packets might be dropped leading again to low packet delivery ratios.

\section{- Greedy Perimeter Coordinator Routing (GPCR)}

In [55] the authors tackle the planarization problem by considering urban streets as a planar graph. Each road segment represents an edge of the network topology graph, and the road junctions represent the vertices. This is because vehicular traffic density is highly variable with space and time, and it is impractical to create planar graphs in GPSR protocol. In this representation of the urban map, nodes forward data packets in greedy and perimeter mode until they enter the intersections.

At Intersections, nodes make actual routing decisions concerning which next road segment is the best option for packet routing. Therefore, packets should be forwarded to a node on the junction, which is known as the coordinator node. This is illustrated in Fig. 10a, where node $\mathrm{x}$ forward packets to any nodes $\mathrm{y}$ in the corner (coordinators). These nodes usually have more alternative options to forward packets.

The authors proposed two heuristics to determine whether a node is a coordinator or not. The first one uses beaconing services so that each node is aware about its neighbors. A node can be considered a coordinator node when it has two neighbors that are within radio range of each other, but do not list each other as a neighbor. This case is demonstrated in Fig. 10b, where nodes $\mathrm{x}$ and $\mathrm{z}$ are neighbors of node $\mathrm{y}$, but they do not list each other as neighbors. This method has problems, as demonstrated in Fig. 11. Since node $y$ is located on a curve, it cannot be considered a coordinator node. The second one 


\section{Macrothink}

is derived by calculating the correlation coefficient that relates a node to its neighbors. A correlation coefficient close to zero indicates that there is no linear coherence between the positions of the neighbors. This indicates the node is located at a junction.
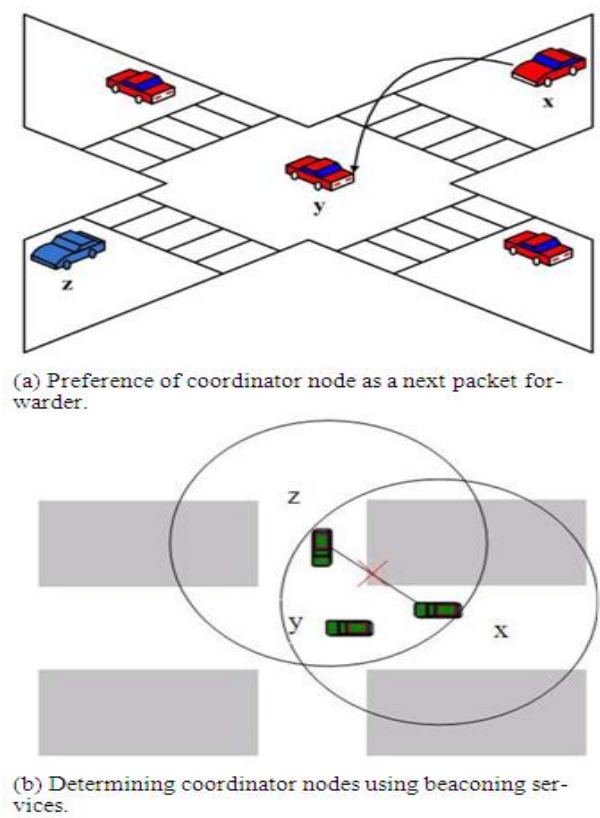

Fig. 10: Packet forwarding mechanism of GPCR protocol.

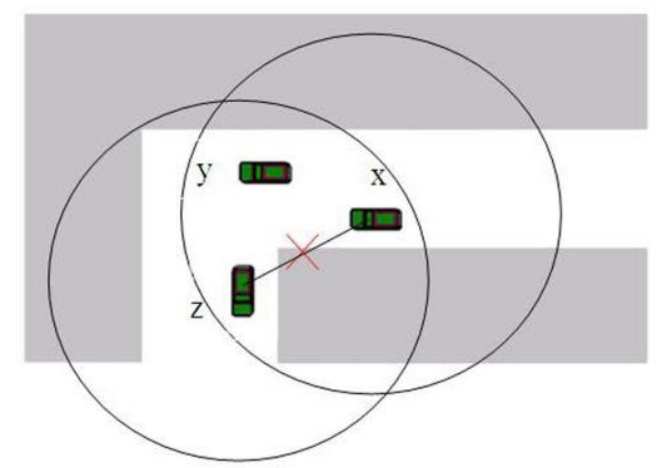

Fig. 11: Discovery failure of coordinator node in GPCR [57].

Performance evaluation shows that GPCR delivers more data packets compared to GPSR. However, there is the possibility that packets loop back in the same street from which the packet has arrived.

- Greedy Perimeter Stateless Routing Junction+ (GpsrJ+)

In [56], Lee et al. (2007) proposed GpsrJ+ to tackle unneeded stop of the coordinator nodes at the intersections in GPCR routing protocol. GpsrJ+ utilizes two-hop beaconing to predict the next road segment in which the packet should be forwarded toward a destination. If the packet carrier node has the same direction as a coordinator node, the prediction mechanism bypasses the intersection and forwards the packet to the node ahead of the junction node. However, if the coordinator node has a different direction than 


\section{NI Macrothink}

the packet carrier node, it selects the coordinator node as a next relay hop. In their performance evaluation, they show that GpsrJ+, in comparison with GPCR and GPSR, increases packet delivery ratio and reduces the number of hops in the perimeter mode of packet forwarding.

\section{- Connectivity-Aware Routing (CAR)}

CAR [58] is a geographical routing protocol designed to tackle the issues of route connectivity between source and destination. CAR uses the idea of AODV routing to find a path to the specified destination. This is by transmission of the route discovery packet to determine at least one path between the source and the destination. The destination responds to the source by sending the route reply packet which includes its position. In $\mathrm{CAR}$, in contrast to AODV, the traversed route request and reply packets record the anchor points along the path of the destination. The anchor points are nodes near the intersections. The velocity vector of these nodes is not parallel to the velocity vector of the node in the packet. In addition, due to route request broadcast, multiple paths might exist in the route request packet between source and destination, but destination selects the one with better connectivity and lower end-to-end delay. Once the shortest path is established toward destination, it forwards data packets geographically through a sequence of anchor points. The list of anchor nodes is included in each forwarded data packet. CAR also proposes the concept of "guards" to help to append the current position of a destination. A guard node can add geographical information to the received packet that will eventually deliver this information to the destination.

The results of performance evaluation have shown that CAR is dominant compared with GPSR in terms of packet delivery ratio. However, broadcasting route discovery in high mobility environments lead to scalability issues. Likewise, each data packet should include a sequence of junction nodes, which again makes the protocol un-scalable.

\section{- Static Node-Assisted Adaptive Routing Protocol (SADV)}

The SADV [59] is a multi-hop geographical routing protocol to forward data packets in vehicular scenarios. The SADV utilizes static nodes to route packets at the intersections in sparse traffic density. These anchor nodes cache packets in the buffer and opportunistically forward it once a vehicle on the best delivery path appears. Between intersections, the data source greedily forwards packets toward the next coming intersection, and each anchor re-computes the best delivery path for the forwarded packets.

Global network information is transmitted to all anchor nodes to improve the accuracy of the information utilized by them when re-calculating the quality of delivery routes. This SADV characteristic may lead to scalability issues due to large bandwidth overhead in the network.

- Anchor-based Street and Traffic Aware Routing (A-STAR)

A-STAR is proposed in [60] to provide end-to-end connection between vehicles in sparse 


\section{Macrothink}

vehicular scenarios. The information of the city bus routes have been used to find the best anchor path with high packet delivery. This anchor path guarantees end to end delivery even in low vehicular traffic densities. When a packet reaches a local optimum, it switches to recovery mode by finding new anchor paths towards the packet destination. The simulation based study has proven its superiority in comparison with GSR and GPSR. However, since the routing path follows the anchor path, it may not be optimal. As a result, it leads to large route delay.

\section{- VANET Cross Link Corrected Routing Protocol (VCLCR)}

In [61], Lee et al. proposed a geographic routing protocol that removes the cross links induced by the perimeter traversal of GPCR protocol. They utilize the idea of loop back packets as a cross-link detection probe. In perimeter mode of packet forwarding, the packet records the route information. This information is useful to check whether the packet is routed back to the starting point. If so, it means there is a routing loop and cross link. Packet forwarding without cross link and loop back, VCLCR performs the same as GPCR. VCLCR increases the successful packet delivery ratio compared with GPCR because of route loop elimination on the packet path.

\section{- Landmark Overlays for Urban Vehicular Routing Environments (LOUVRE)}

In [62], Lee et al. proposed a geo-proactive routing solution, known as LOUVRE, to efficiently route data packets between grids in the urban vehicular environments. The proposed routing solution assumes that all nodes are equipped with a navigation system so that they have knowledge of the city map. With the help of the navigation system, landmark nodes at the intersections and estimated density of the urban roads, LOUVRE can make efficient routing decisions inside a grid or between them. More specifically, it considers the density threshold of the road segments (overlay link) at the time of route establishment on the top of overlay urban environment. Fig. 12 shows the concept of LOUVRE, based on the peer-to-peer traffic density estimation.

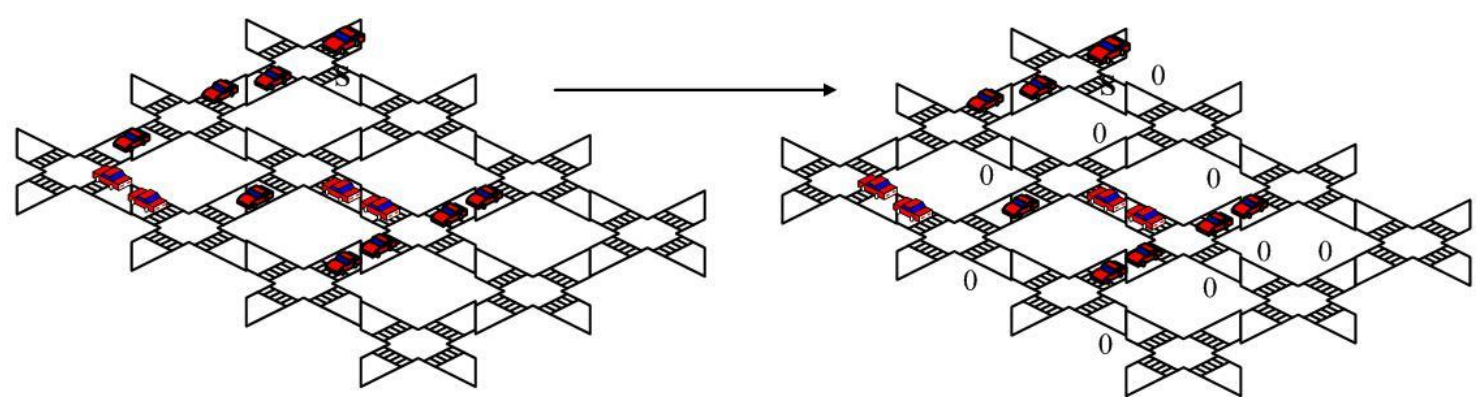

Fig. 12: LOUVRE route establishment

It can trickle the road segments that do not have any traffic density over the threshold, which is determined by the following equation 2. Then Dijkstra's shortest path algorithm can determine the minimum delay route by considering only road segments with higher vehicular connectivity. Thus, the shortest path is automatically established between 


\section{IMacrothink}

source (S) and destination (D).

$$
\text { Density }-\mathrm{Th}=\frac{\mathrm{L}}{2 \cdot \mathrm{R}}+1
$$

where $\mathrm{R}$ is the radio range and $\mathrm{L}$ is the road length. The authors implemented the proposed protocol in Qualnet, and compared it with benchmarks GPSR and GPCR protocols using realistic VanetMobiSim mobility model [63]. Due to efficient routing in overlay and underlay networks, LOUVRE offers better packet delivery ratio, hop count, and packet delay. Obviously, however, this protocol does not scale well with increased network size due to its peer-to-peer traffic density estimation, as this method requires nodes to have global knowledge of the whole network graph.

\section{- $\quad$ Road-Based using Vehicular Traffic Routing Protocol (RBVT)}

A positive step toward efficient routing protocols is taken by the authors in [64], where the authors proposed RBVT, which leverages on-board navigation systems to establish paths between the source and the destination through a sequence of intersections with high network connectivity. Furthermore, geographical forwarding is proposed to forward data packets between two consecutive junctions on the path. RBVT's route discovery and route reply is similar to the CAR protocol. RBVT, however, uses real-time vehicular traffic information so as to make nodes aware of the city map. This increases RBVT's robustness and adaptability to network conditions. Besides, since it considers road-based paths and geographical forwarding, the selected route should be stable. This class of routing protocol consists of two different protocols based on routing demand and these are known as reactive and proactive protocols. Reactive protocol (RBVT-R) makes route discovery decisions on demand (like reactive topology-based routing protocols) and reports to the source with a route reply which includes a list of traversed junctions. RBVT-P creates and maintains the route pro-actively by transmission of periodic Connectivity Packets (CPs). These packets visit connected road segments and cache the topology that they traversed. All nodes utilize this information to determine the shortest path to the destination. Fig. 13 shows the main concept of both classes of RBVT routing protocol. The RBVT creates a path (S, I1 , I2, I3 , I4, D), whereas the shortest path solutions forward data packets through a route $(\mathrm{S}, \mathrm{I1}, \mathrm{I} 3, \mathrm{I} 4, \mathrm{D})$ that would lead to route break.

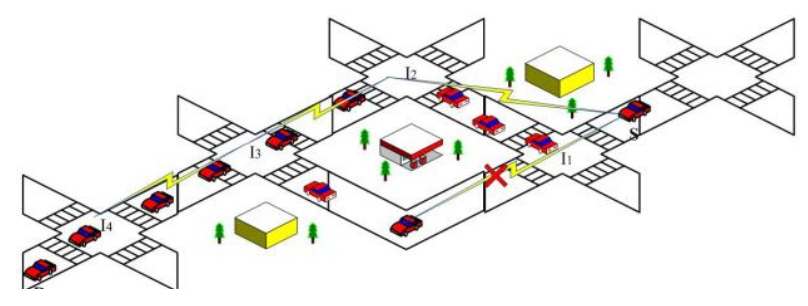

Fig. 13: RBVT routing concept

In addition, to eliminate the hello packet, the authors proposed an enhancement of receiver-based next hop self election (e.g., [65], [48] or [49]) to reduce protocol overhead 


\section{MInstitute Macrothink $^{\text {Int }}$}

in the network. However, exchanging link state information and route maintenance leads to high network overhead. In [64], the authors use beaconless forwarding optimization between intersections. In their forwarding optimization, they used power signal strength, optimal transmission range and distance parameters for packet forwarding.

In the performance evaluation, the results show that both classes of RBVT perform better than each of the AODV, GSR, OLSR and GPSR. Furthermore, the RBVT-P is more reasonable for delay-sensitive applications, whereas RBVT-R can be used for applications that require high throughput. However, since RBVT requires the exchange and maintenance of non-local information, it leads to high network overhead. Data packet headers carry a list of junctions that the packets should follow. Similar to CAR, it might lead to un-scalability issues. Furthermore, in their optimized geographical forwarding, the direction of vehicles is not taken into consideration.

- Improved Greedy Traffic-aware Routing Protocol (GyTAR)

Another direction to forward data packets optimally over urban vehicular environments is taken by [66] where the authors proposed an improved vehicular ad hoc routing protocol for city environments (GyTAR). The designed protocol has two modes of operation: routing at the intersections and at road segments. For the former mode, GyTAR reactively selects neighbour intersections upon consideration of variations in traffic density and the distance to the destination. For the latter, GyTAR uses greedy routing to forward data packets. The concept of GyTAR's intersection selection is demonstrated in Fig. 14. As can be seen, once the source node reaches intersection I1, it scores junctions I2 and I3 based on traffic density and their proximity to the destination. GyTAR, then, selects intersection I 2 because it has higher connectivity than I1.

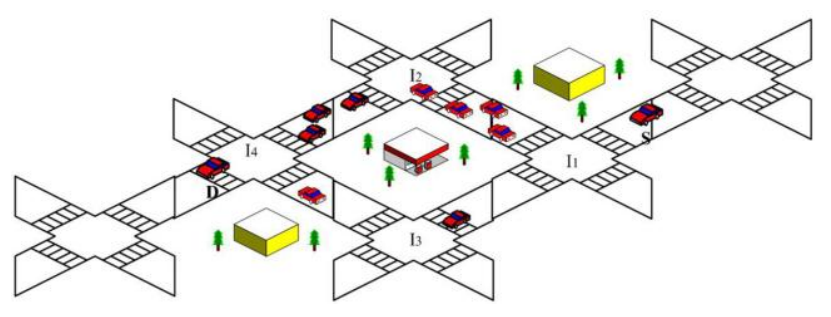

Fig. 14: GyTAR's intersection scoring

After GyTAR is implemented and compared with GSR and Location-Aided Routing (LAR) [67], the results show that GyTAR is superior in terms of packet delivery ratio, packet delay and routing overhead. However, since realistic city maps have irregular shapes such as unequal road segments between intersections, GyTAR does not consider variations of segment lengths within urban environments. Furthermore, GyTAR's distributed local density estimates are based on clusters. Only the cluster head Cell Density Packet (CDP) arrives at the intersection. Since VANET is a high mobility network, maintenance of the clusters is hard and is on the expenses of network bandwidth.

- Diagonal-Intersection-based Routing (DIR) 
The authors in [68] proposed DIR protocol for routing packets in urban vehicular scenario. The DIR protocol consists of three phases- destination discovery, packet forwarding, and route maintenance- to route packets efficiently towards the destination. The simulation results from the performance evaluation show that the proposed routing protocol, compared to CAR, can improve end-to-end packet delay, packet delivery ratio, and network throughput. However, the periodic maintenance of link cost (expected packet forwarding delay) between for-warding diagonal intersections (from $\mathrm{I}_{\mathrm{XI}, \mathrm{YI}}$ to $\mathrm{I}_{\mathrm{XJ}, \mathrm{YJ}}$ ) leads to bandwidth overhead traffic and hence negatively creates an impact on the end-to-end data transfer performance. The protocol is also susceptible to un-scalability issues, not just due to the bandwidth overhead of finding link costs, but also on finding a list of anchor points between source and destination.

\section{- $\quad$ TOpology-assisted Geo-Opportunistic Routing (TO-GO)}

In [69], Lee et al. proposed TO-GO, which is a geo-graphic routing protocol that exploits local information of 2-hop neighbors via beaconing to select the best target forwarder and incorporates opportunistic forwarding with the best chance to reach it. The authors divided TO- GO into three algorithms: Next-hop Prediction Algorithm (NPA), which determines the target node in the same road segment of the data source, the Forwarding Set Selection algorithm (FSS) determines the nodes that are contributing in the forwarding set and priority scheduling algorithm selects the best candidate node (which is closer to the target node) in the forwarding set.

In the performance evaluation, the proposed TO-GO is compared with GPSR, GPCR and GpsrJ+ using Vanet-Mobisim mobility model and log-normal shadowing radio propagation model. In ideal wireless channels between vehicles, the result shows TO-GOs performance comparable to GpsrJ+ while GPSR and GPCR lag behind. In realistic erasure wireless channel, TO-GO performs better than GpsrJ+ in terms of the packet delivery ratio.

\section{- Fuzzy Logic-based Route Selection in VANET}

In [70], Huang et al., proposed a load balancing and congestion avoidance routing mechanism (named Fuzzy Logic (FL) and Fuzzy Logic H-Infinity Filtering (FLHF)) over short radio ranges to guarantee the Quality of Service (QoS) requirements of real time traffic. A fuzzy logic decision making system is utilized to select the intermediate nodes on the routing path through inter-vehicle communication. The simulation based study revealed that the proposed mechanism obviously achieves excellent performance in highway traffic scenarios. While this mechanism has been developed to deal with load balancing and congestion avoidance issues, it does not consider unreliable wireless channels and disconnectivity issues in VANET.

In addition, the authors in [71] propose Fuzzy control based AODV routing (Fcar) for highway vehicular scenarios. In the proposed protocol, the authors utilized a group of vehicles and route lifetimes as routing metrics for rebroadcasting decision making processes. However, since the topology-based routing protocols [2], [3] are less preferable in high speed environments (due to high protocol overhead), these routing protocols embed 
fuzzy decision making systems with reactive address-based routing.

In another attempt, in [72] we developed a novel De-lay and Reliability aware geographical Routing $\left(\mathrm{DR}^{2}\right)$ protocol that selects a low latency, high reliability and shortest path toward a destination. The $\mathrm{DR}^{2}$ utilized cross layer communication between Medium Access Control (MAC) and network layer. In essence, the MAC layer observes the Signal to Noise ratio (SNR), delay and velocity vector difference metrics for all paths of neighbor nodes, network layer then could select the best preferable path through the fuzzy inference system. The H-infinity technique was used to optimize membership functions with respect to the volatile characteristics of VANET.

In [73], the authors proposed a fuzzy-assisted social-based routing (FAST) protocol that takes the advantage of social behavior of humans on the road to make optimal and secure routing decisions. FAST uses prior global knowledge of real-time vehicular traffic for packet routing from the source to the destination. In FAST, fuzzy inference system leverage friendship mechanism to make critical decisions at intersections which is based on prior global knowledge of real-time vehicular traffic information. The simulation results in urban vehicular environment for with and without obstacles scenario show that the FAST performs the best in terms of packet delivery ratio, average delay and hops count compared to the state of the art VANET routing solutions.

\subsubsection{Hybrid (Packet and Non-Packet Buffering) Geographical Routing Protocols:}

The aforementioned geographical routing protocols, such as GPSR and GPCR, route data packets by using the greedy algorithm concept. That is, the data source selects the next hop which has more advanced progress to-wards the destination, or utilizes recovery algorithms in case such algorithms fail. These geographical routing solutions are efficient and could route data packets when there are enough nodes in the vehicular scenario. In other words, they fail when there is a topological hole between the packet carrier node and its neighbors. The notable hybrid geographical routing proto-col is the Geographic DTN Routing with Navigator Prediction for Urban Vehicular Environments [74]. In GeoDTN+Nav, the authors combined greedy mode, perimeter mode, and DTN mode. The GeoDTN+Nav protocol utilizes a network partition detection method so as to switch between different modes of packet forwarding. Network partition detection switches between different modes based on the number of hops a packet has travelled so far and the delivery quality of neighbors. The Virtual Navigation Interface (VNI) has been used to provide necessary information for the proposed protocol so that it can determine its routing mode and next hop forwarder.

In performance evaluation, the proposed protocol delivers more packets in comparison with GPSR and GPCR in sparse or partitioned networks. However, this is at the expense of packet delay in dense traffic conditions.

In addition, in [75] we proposed a novel Stability and Reliability aware Routing (SRR) protocol that forwards packets with a high degree of reliability and stability towards the destination. That is, the SRR protocol incorporates fuzzy logic with geographical routing when making packet forwarding decisions. Routing metrics, such as direction and distance, are considered as inputs of the fuzzy decision making system so that the best preferable 
neighbor around a smart vehicle is selected. We then utilize a mechanism to cache data packets once the network is disconnected and then switch back to SRR in a connected vehicular scenario. Traffic density is considered as an input when estimating network dis-connectivity. After developing an analytical model of our protocol, we implemented it and compared it with standard protocols. In a realistic highway vehicular scenario, the results show that the SRR protocol performs better than GPCR and DSR in terms of packet delivery ratio, packet delay and control overhead.

\section{Influence of Mobility Model}

Performance evaluation in VANET requires the interaction of network access components and mobility model simulator. In vehicular networks, the mobility model is used to simulate vehicles movement in the urban/highway roads. This simulator will convert the movement of vehicles to a mobility trace file, then the trace file can be fed to a vehicular network simulator for performance evaluation of VANET applications [76]. As demonstrated in [77] and [78], the simulation results are significantly affected by the chosen mobility model simulator. This is because the vehicle's movement traces heavily influence t he network connectivity and hence network performance. Thus, realistic movement traces are necessary to evaluate the VANET protocols [79].

In [76], the authors compared the simulation results of AODV and GPSR based on random way-point mobility model with realistic vehicular movement traces. In most cases, they observed that the performance of both routing protocols is strongly dependent on the mobility model. More precisely, when the mobility model is random way-point, AODV always lags behind the GPSR. By using vehicular movement traces, on the contrary, AODV always performs better than GPSR. Based on this study, mobility model has significant affect on the simulation results of routing protocols.

TABLE II: Simulation parameters

\begin{tabular}{|l|l|}
\hline Parameters & Value \\
\hline Simulation time & $350 \mathrm{~s}$ \\
\hline Simulation area & $3968 \mathrm{~m} \times 1251 \mathrm{~m}$ \\
\hline Mobility model & STRAW \\
\hline Traffic Density & $100-400$ nodes \\
\hline Vehicle velocity & $30-60 \mathrm{~km} / \mathrm{hr}$ \\
\hline Transmission range & $250 \mathrm{~m}$ \\
\hline Maximum packet generation rate & 12 packet/second \\
\hline Maximum number of source nodes & 10 \\
\hline Transmission data rate & $3 \mathrm{Mbps}$ \\
\hline MAC protocol & IEEE $802.11 \mathrm{p}$ \\
\hline Data packet size & 512 bytes \\
\hline
\end{tabular}




\section{Ml Macrothink}

\section{Performance Evaluation}

This section presents the evaluation of the representatives of geographical and topology-based routing protocol in urban vehicular environments. We have simulated the standard protocol using the packet level simulator JIST/SWANS [80]. It is de-signed based on the TCP/IP five layer network communication architecture. The simulation scenario is $3968 \times$ $1251 \mathrm{~m}$ area that was configured with JIST/SWANS, and the selected area contains 370 road segments (Fig. 15). We used the open source STreet RAndom Way point mobility model (STRAW) [81] to simulate the movement of vehicles. STRAW has an efficient car following trajectory, lane changing model and real-time traffic controller. In STRAW, the generated vehicles are distributed regularly in the urban streets, and they pause for a period of time at the intersections.

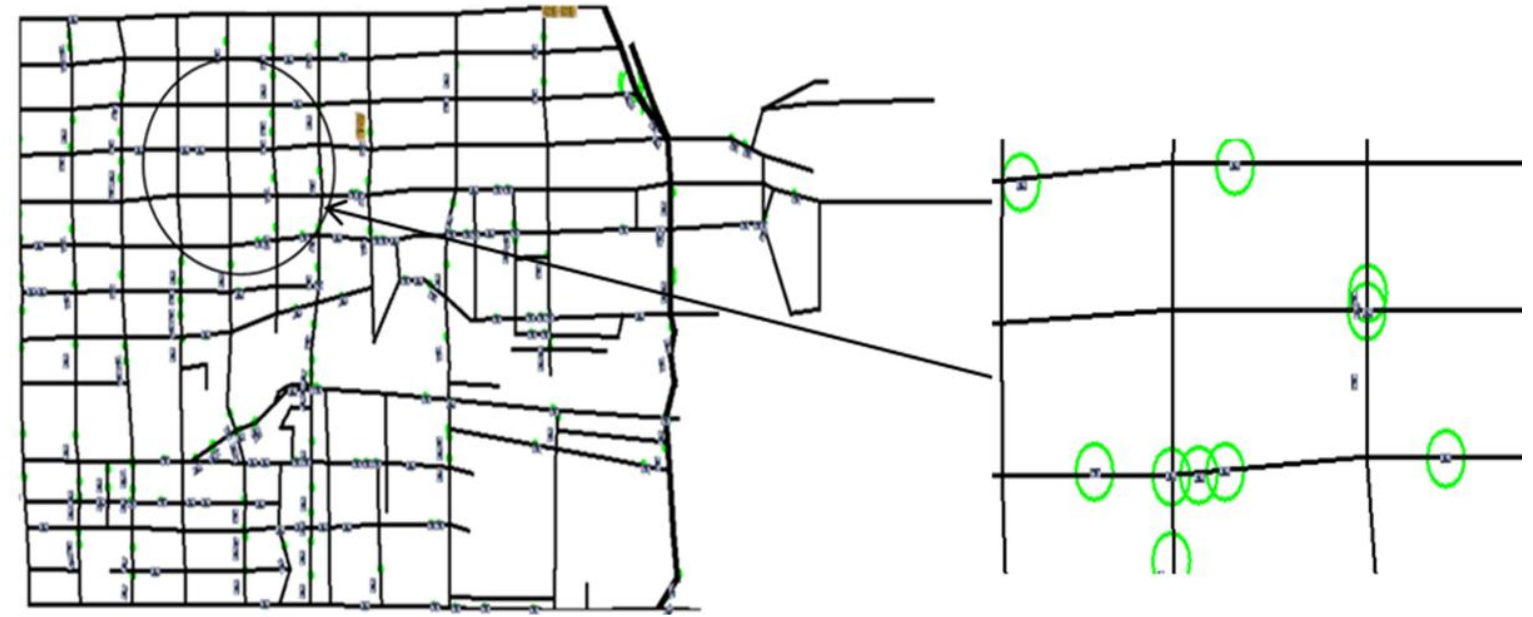

Fig. 15: A snapshot of Chicago city environment during simulation

Fig. 16 illustrates the actual map of the Chicago city.

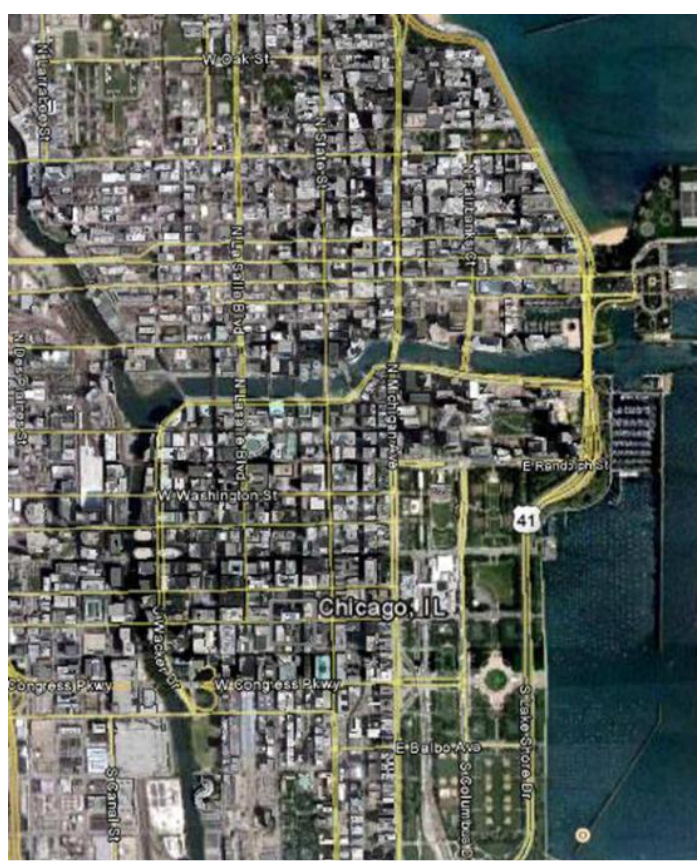

Fig. 16: Map of the region of Chicago city used in the simulation scenario 
In addition, at the physical layer, the shadowing channel model has been used to characterize the wireless channel [82]. In the simulation, the value of the path loss exponent $\mathrm{n}=2.8$ and the reference distance $\mathrm{d} 0=0.4$ are used for the shadowing model [71]. Furthermore, we set the radio communication range at 250 meters. In the simulation area, the traffic density of vehicles is varied from 100 to 400, and they move along the roads with an average speed ranging from 30 to $60 \mathrm{~km} /$ hour. Moreover, the IEEE 802.11p standard is used to model MAC layer. The simulation key parameters are summarized in Table II. The selection of these simulation parameters is based on the studies [83, 84, 85, 71]. This is because these studies were based on the realistic measurements between nearby vehicles. Further, the total simulation time is 350 seconds. We set the settling time to 25 seconds at the beginning of simulation to remove the effect of transient behavior on the results. The total simulation time also included 25 seconds of stop sending packets from the end of the simulation. It is worth mentioning that each point in the performance figures exemplifies the average of 20 simulation runs.

Accuracy of simulation results significantly reflected the credibility of the data from a specific measurement. Validation is used to evaluate the performance gain which is obtained from the proposed solution. More precisely, statistical significance test, namely Analysis Of Variance (ANOVA-single factor) was calculated to verify the measured data form a specific protocol. ANOVA is a statistical analysis model which is used to partition the variance of a particular variable into components which are attributable to different sources of variation.

In the comparison study, we have compared the performance of the representatives of geographical routing protocols (GPCR) [55], Stability and Reliability aware Routing (SRR) in [75], (CBF) [48] as well as topology-based (AODV) [2] routing protocols.

We now briefly review the basic operation of these routing protocols: GPCR is a geographical routing protocol that for-wards packets to a neighbor node which has the closest distance to the destination (greedy mode of packet forwarding). In the perimeter mode, a node forwards packets to the next neighbor node by applying right hand rule. In addition, GPCR assumes that the road traffic is the planar graph, which utilizes the concept of junction nodes to control the next road segments that packets should follow; CBF uses the distributed timer-based mechanism for the data packet forwarding decision. This random timer mechanism is set when the relay nodes receive the RTS frame and check if they are closer to the destination than the packet carrier node. The contention between relay nodes will end as one of them responds the source by sending CTS frame (Which is a contention winner and selected as the next hop); SRR protocol forwards data packets with a high degree of reliability and stability towards the destination. The SRR protocol uses fuzzy inference system to make packet routing decisions. Routing metrics, such as direction and distance, are considered as inputs of the fuzzy decision making system so that the best preferable neighbor around a smart vehicle is selected. Moreover, the SRR protocol exploits caching mechanism to salvage data packets once the network is disconnected and then switch back to SRR in a connected vehicular scenario. Traffic density is considered as an input when estimating network dis-connectivity. 


\section{Macrothink Institute ${ }^{T M}$}

The following metrics are used for the performance 3valuation:

1) Packet Delivery Ratio (PDR): measures the fraction of data packets that are successfully received by the destination to those generated by traffic source.

2) End to end delay: is the total time required by all the packets to travel from the source to the destination. The packet delay obtained in the simulation is the sum of sending buffer, medium access (packets delay due to interface queue), re-transmission, relay election and propagation delay.

3) Hop count: is the average number of relay nodes that forward data packets to the destination.

In the performance evaluation, we conducted different experiments to study the effect of various parameters on the representative of the routing protocols.

\subsection{Impact of Node speed}

This study is performed with a traffic density of 300 nodes with 10 of them acting as a source. To investigate the effect of speed on the performance of the routing protocols, we run the experiments with varying the mobile speed from $30 \mathrm{~km} /$ hour to $60 \mathrm{~km} /$ hour. The simulated beacon interval is $0.5 \mathrm{~s}$ for the studied (SRR, GPCR and AODV) protocols. Fig. 17 shows the packet delivery ratio of the SRR, GPCR, CBF and AODV protocols with respect to vehicle speed. A prompt result of this performance evaluation is that an increase in vehicle speed leads to a low successful packet delivery ratio for all protocols. In more detail, as can be seen, the $\mathrm{CBF}$ protocol is far superior compared with the other routing protocols. The reasons are that the $\mathrm{CBF}$ protocol removes the beacon messages to update the neighbor information, which leads to less bandwidth consumption in the network and the required memory to store neighbor information. As a consequence, the percentage of the link utilization will increase for data packet transfers.

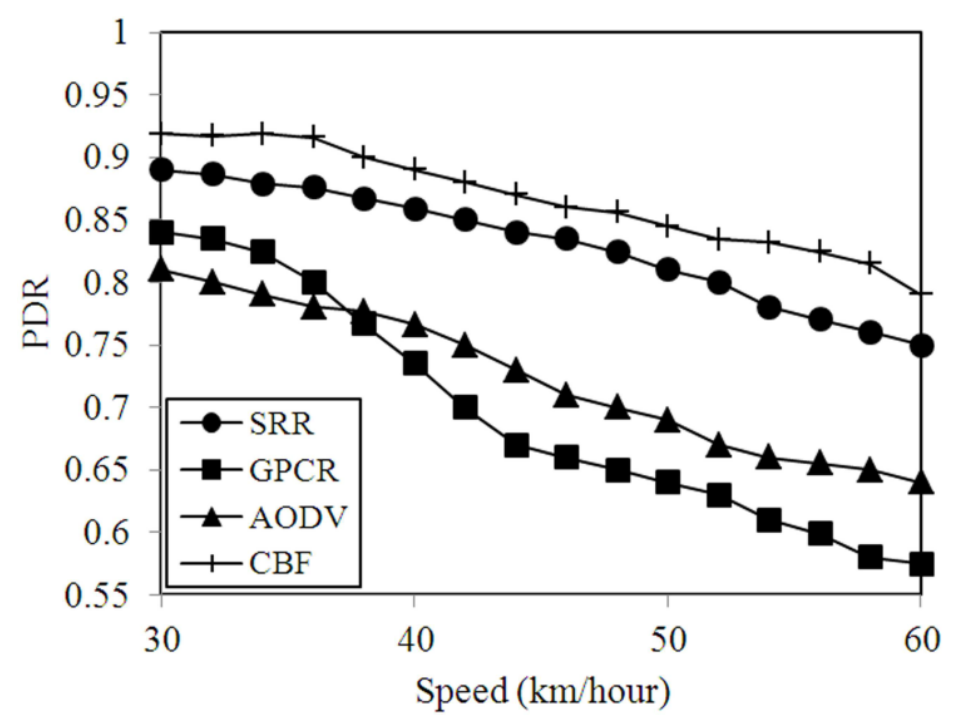

Fig. 17: Packet delivery ratio with respect to vehicle speed for SRR, GPCR, CBF and AODV protocols. 
In contrast to beaconless forwarding protocol, in GPCR protocol, the packet carrier node needs to know the position information of all direct neighbors. This information is obtained through periodic beacon messages sent out by each direct neighbor node. The high mobility of vehicles leads to the staleness of neighborhood information. As a result, the trend of GPCR protocol acutely drops to $57.5 \%$ at a speed of $60 \mathrm{~km} /$ hour. On the contrary, we observe that the SRR protocol is always performs better than the GPCR protocol. This is not surprise since GPCR protocol only uses greediness factor as a routing metric to forward data packets in such unreliable and unstable vehicular scenario while SRR protocol favors more stable and reliable links as well as forwarding progress toward the destination. Consequently, the SRR protocol maintains the trend of average PDR to $82.9 \%$. Moreover, the AODV protocol also degrades rapidly because the established routes between source and destination break frequently, and the source node should perform a route discovery or the en-route nodes should send route error notifications to the source node. This leads to a steep decrease of packet delivery rate $(64 \%$ at $60 \mathrm{~km} /$ hour$)$.

In addition, even though each direct neighbor node utilizes its own accurate location information, the CBF protocol suffers slightly when mobility increases to $60 \mathrm{~km} / \mathrm{hour}$. We believe that this is because the elected direct neighbor node will exit the radio range before receiving the actual data packets or sends back the CTS frame to the source.

ANOVA single factor has been used to compare the means of the state of the arts routing protocols. The result indicates that the $\mathrm{CBF}$ protocol has the lowest variance compared with the other routing protocols. The variance of SRR, GPCR, AODV and CBF are 0.002186814, $0.008685963,0.00347865$ and 0.001703563 respectively for PDR with $F$ value of 27.16494221 and $\mathrm{P}$ less than $1 \%$ level of significance. These results suggest that the SRR protocol has lower variance than other three protocols. The implication is that the CBF protocol may be more efficient in increasing PDR in the urban vehicular scenario than the other three routing protocols as it shown in Fig. 17. Thus, the applied ANOVA single factor validation method is significantly reflect credibility of the variance o f data from specific measurement of the proposed protocol.

In Fig. 18, we show the effect of increasing vehicle speed on average packet delay. The $\mathrm{CBF}$ protocol has the smallest average delay among the protocols studied. In CBF protocol, the route is determined based on the modified RTS/CTS frames handshaking and this mechanism is more effective in reducing the traffic load on the MAC layer. This leads to improved delays, because fewer retransmissions and exponential back-offs happen in the MAC layer. Contrarily, in GPCR, the average delay increases drastically with higher mobility. This is because the number of MAC layer retransmissions increases. Furthermore, the delay trend of AODV is also ascending steeply (2.19 s at $60 \mathrm{~km} / \mathrm{hour}$ ) because high mobility leads to frequent route breaks between the source and the destination. 


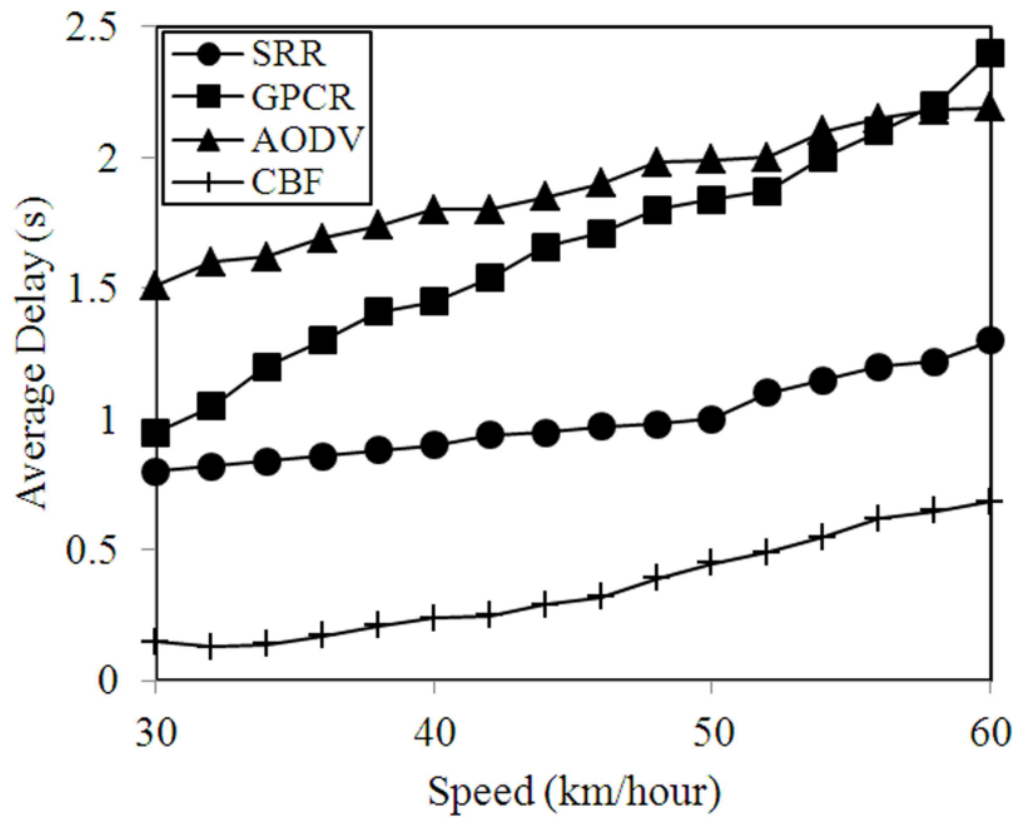

Fig. 18: Average packet delay with respect to vehicle speed for SRR, GPCR, CBF and AODV protocols.

In addition, we observe that the GPCR protocol suffers in terms of average delay as compared to the SRR protocol. This can be attributed to the fact that the source node in GPCR selects a neighbor vehicle based on greediness factor; that is, a relay node which has shortest distance to the destination will be elected as a next packet forwarder. Only considering greediness factor for packet forwarding leads to data packet losses in unreliable wireless channels between vehicles. As a result, MAC layer tries to perform redundant retransmissions to compensate these data packet losses. With these packet retransmissions, the GPCR protocol is susceptible to higher end-to-end delay. As can be seen in Fig. 18 the delay trend of GPCR increases to $2.4 \mathrm{~s}$ at a speed of $60 \mathrm{~km} / \mathrm{hour}$.

The comparison between the state of the art routing protocols in Fig. 19 indicates that the SRR protocol has slightly longer average path length than GPCR protocol. The reason is that unlike GPCR, SRR protocol explores the paths to the destination by considering link reliability (considering power strength) and link stability of the route toward the destination. Expectedly, the routing protocols should perform better for shorter path lengths. However, the results do not support this hypothesis, because selecting better en-route nodes leads to better performance. For instance, the SRR protocol has a longer path length, but it performs better than GPCR protocol. 


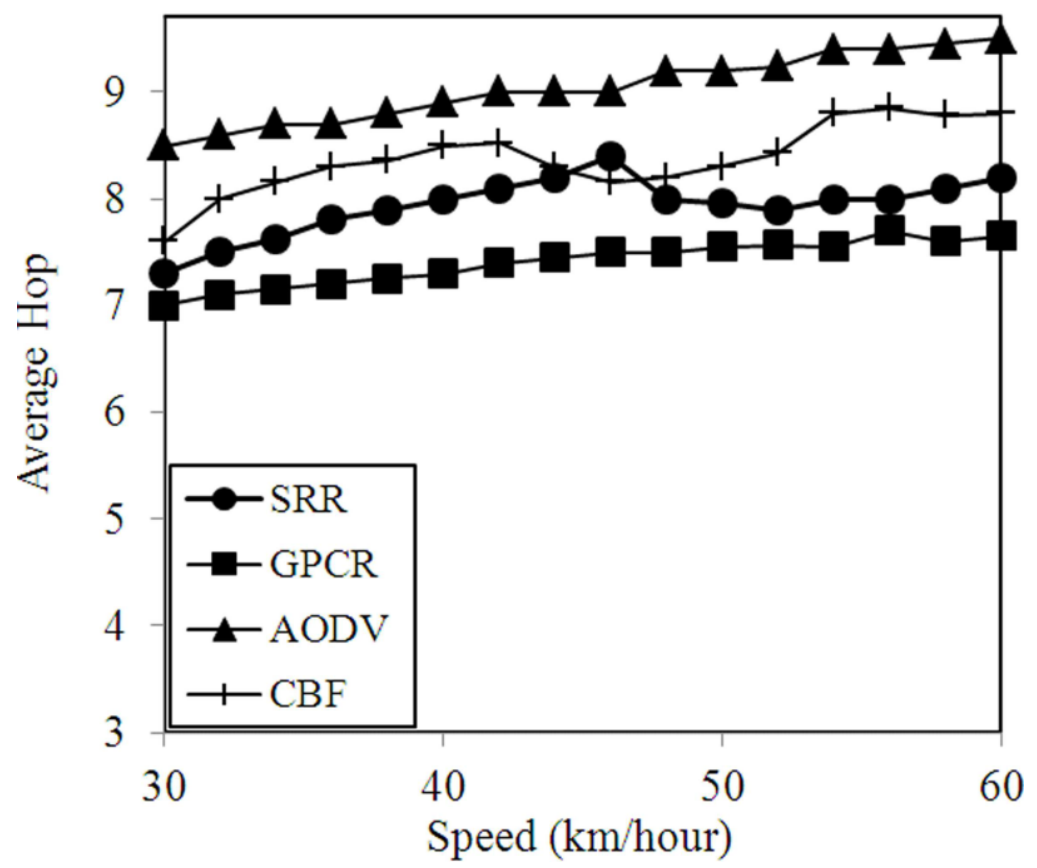

Fig. 19: Average path length with respect to vehicle speed for SRR, GPCR, CBF and AODV protocols.

\subsection{Impact of Traffic Density}

In this study, we conducted experiments to understand the effects of a variable number of vehicles on the performance of existing routing solutions. The experiments involved setting the vehicle speed at $45 \mathrm{~km} /$ hour and the number of source nodes at 10 . We ran the simulation with different number of nodes ranging from 100 to 400 .

In Fig. 20, the trend of the average delivery ratio is plotted with the different number of vehicles. As expected, the trend of protocols show that the successful packet delivery ratio consistently increased as the number of vehicles increases. This is not surprising since the probability of connectivity is increased with the increasing vehicular traffic density. In more detail, the GPCR protocol greedily forwards data packets toward the destination. The link between the packet carrier node and the selected next hop will be very weak (move out the radio range). This case leads to fewer packets delivered to the specified destination. Moreover, in greedy packet forwarding, the probability of link failure increases due to high signal attenuation of unreliable wireless channels. As a result, the network performance is degraded due to high packet loss. Due to these cases, the SRR protocol performs better as compared to the $\mathrm{CBF}$ protocol. But, in comparison to AODV, GPCR protocol tends to perform better due to higher connectivity in the network. 


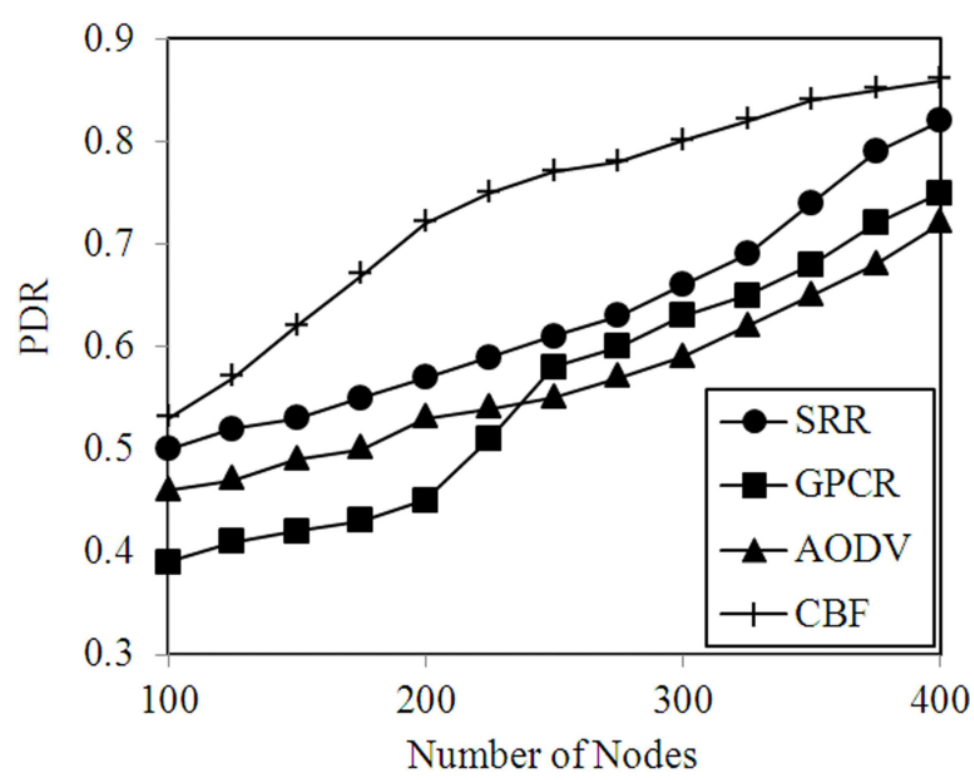

Fig. 20: Correlation between Packet delivery ratio and traffic density for SRR, GPCR, CBF and AODV protocols.

The CBF protocol uses relay node self election mechanism to greedily forward data packets toward the destination. In this type of forwarding, nearby nodes do not exploit beacon frame for handshaking and information exchange. This leads to less overhead in the network and hence more delivered data packets towards the destination. However, when node density is sufficiently high (300 nodes or more), the CBF protocol's trend becomes flat. This is because the RTS/CTS handshaking procedure increases the probability that a packet collision will occur as the packet is routed towards the destination.

Another interesting metric is the average packet delay, which is depicted in Fig. 21. We notice that the average packet delay for CBF protocol consistently decreases until the number of nodes becomes 300 , then rises slightly to $280 \mathrm{~ms}$ at 400 nodes. The reason is that low traffic density in the network increases the likelihood that the network will be dis-connected during the forwarding process, whereas the high traffic density leads to packet collision and duplication. Consequently, in both cases, the average packet delay slightly increases. Similarly, as shown in Fig. 21, the average packet delay trend of SRR increases in sparse and dense vehicular scenarios. In sparse vehicular networks, the responsibility of this packet delay is due to buffering data packets for another forwarding opportunity when the network is disconnected. Dense vehicular scenario, on the other hand, causes higher control overhead within the radio coverage of each node which drives higher end to end delay.

In GPCR, The average packet delay steeply increases with traffic density. There are two reasons for this: First, when the number of nodes increases, the time to determine next packet forwarder (which is close to the destination) also increases. Second, unlike SRR protocol, GPCR does not favor link reliability and stability. In addition, the general upward trend of AODV is due to the fact there is now a connected path which drives the average delay up. 


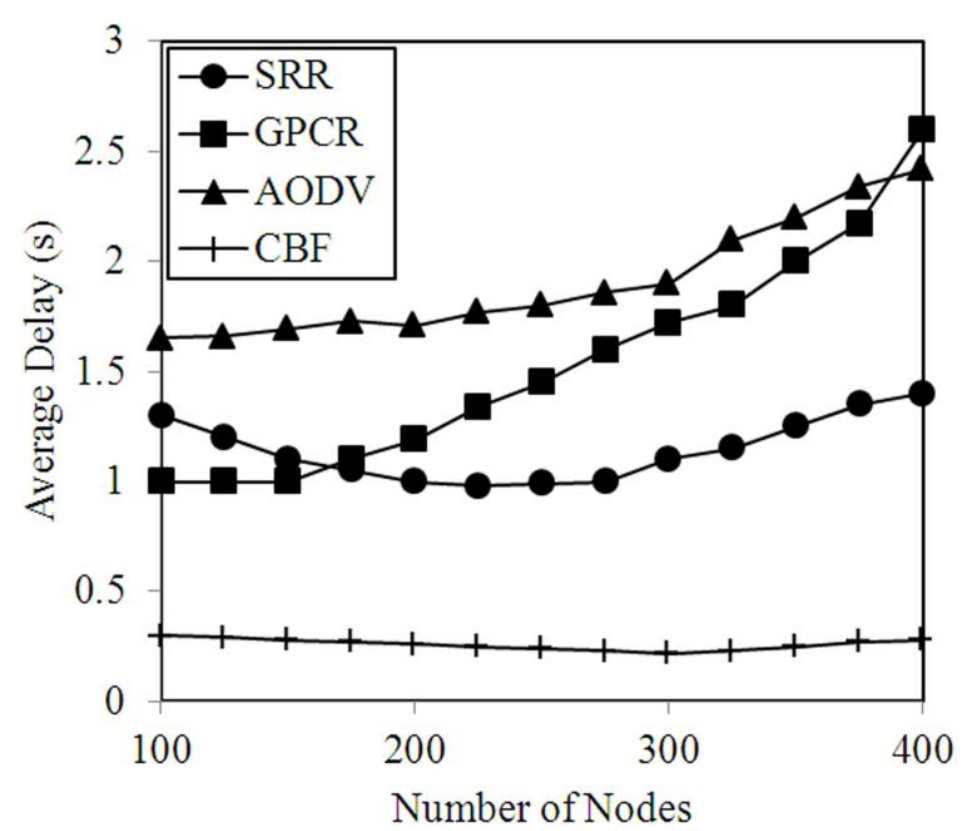

Fig. 21: Correlation between Average packet delay and traffic density for SRR, GPCR, CBF and AODV protocols.

Fig. 22 shows the average path length variation with traffic density. Comparing the hop count incurred by the state of the arts, we notice that the average path length of AODV protocol is slightly longer than that of GPCR, CBF and SRR. However, in comparison to AODV, the SRR protocol offers better performance in terms of successful delivery ratios and average end-to-end delay. The reason for this effect lies in the favoring link reliability and stability in addition to the forwarding progress.

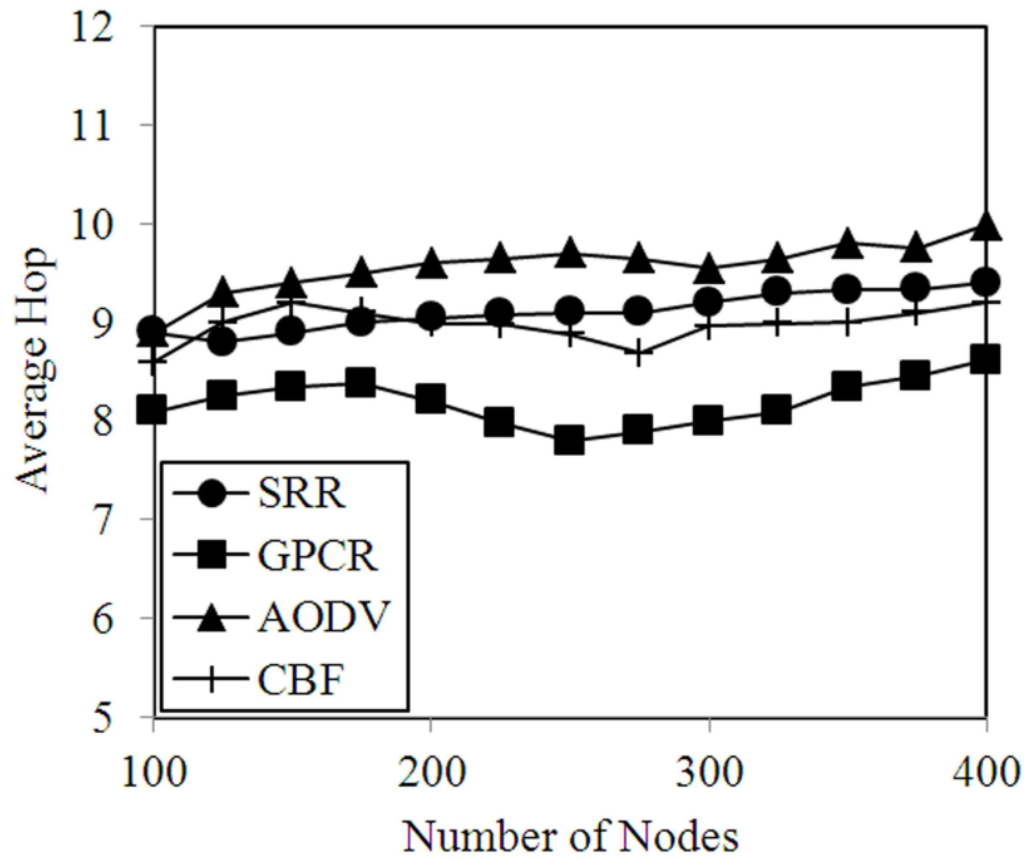

Fig. 22: Correlation between Average path length and traffic density for SRR, GPCR, CBF and AODV protocols. 


\section{Research Directions and Open Issues}

The state of the arts routing solutions is reviewed, compared and criticized. These solutions can be counted as a basement of the routing in vehicular environments. However, yet they do not address many open issues. The following are some of the research directions.

Forwarding optimizations: Most of the reviewed routing solutions considered unit disc transmission range, which does not hold true in the realistic vehicular environment as shadow-ing and different types of fading affects the radio propagation. Thus, it is necessary to utilize packet forwarding optimization by considering Quality of Service (QoS) during packet routing.

Routing in multi-radio enabled vehicular networks: Next Generation Network (NGN) aims to integrate different radio access technologies in order to provide seamless mobility and QoS at anywhere and anytime. Thus, it is crucial to design efficient routing protocols over different wireless access technologies (WiFi, WiMAX, cellular) and decision for optimal selection between them in heterogeneous vehicular networks.

Routing in infrastructure-based vehicular networks: The implementation cost of cellular communication systems is high compared with infrastructure-based vehicular networks. In vehicular networks, the access points can be used as an intermediate node to relay data packets to other vehicles in multi-hop fashion. Thus, designing efficient routing in this environment can be used in various applications such as e-commerce, Electronic Toll Collection (ETC) and road-side advertisement services.

Multimedia routing over vehicular networks: In [42], we recently highlighted the interesting applications of Multimedia (e.g., video and audio) communication over VANET. However, routing of delay sensitive application is very challenging due to a demand of low latency and high reliability of their packet forwarding. We believe geo-proactive routing protocols are the best solution for routing delay sensitive packets [64]. This is because these types of routing store routes' pro-actively in its cache rather than reactively finds a route to a destination.

Multi-hop beaconing in urban environments: In urban areas, there are different types of radio obstacles such as trucks (moving obstacles) and buildings (static obstacles) where single hop beaconing can not penetrate them. Thus, multi-hop beaconing might be necessary in non-line of sight areas.

Traffic-aware and network-aware beaconing approaches: Beaconing frequency adaptation is a challenging future research work. The vehicular traffic situations and network load might be considered to tune the duty cycle of beaconing generation. In this case, the compromise of beaconing load and accuracy of positioning requirements should be taken into consideration in response with heterogeneity of vehicular environments.

Realistic Vehicular Network Scenarios: As witnessed from the state of the arts described in earlier sections, most of the novel routing and beaconing solutions were simulated in unrealistic vehicular scenarios, i.e., a vehicular scenario without considering short-term and 
long-term fading. The difference between simulation experiments using realistic and unrealistic vehicular topologies may result in the expense of human lives which is not affordable. Therefore, the existing research works need to be validated using realistic vehicular scenarios.

\section{Conclusions}

In this article, we have detailed several geographical routing protocols that either specifically proposed or adapted for the vehicular environments. The summary is presented in table III. The performance comparison we pointed out was confirmed by the simulations based on the routing metrics such as packet delivery ratio, packet delay, average path length, throughput and routing overhead. The existing research showed poor performance for the topology-based routing, specifically proactive solutions due to local cache maintenance. The geographical routing protocols are efficient in high speed vehicular networks and each of them is designed for specific condition or routing issue. Furthermore, simulations using a realistic city scenario have shown that the beaconless (CBF) and delay-tolerant (SRR) routing protocols performs better in terms packet delivery ratio and average packet delay as compared to the AODV and GPCR routing protocols. For routing protocols, there is no a unified solution or a standard benchmark for performance evaluation. Thus, solving the aforementioned issues and developing a benchmark routing solution for evaluation purpose are worth the effort in the future research opportunities.

TABLE III: Summary Table of Routing Protocols

\begin{tabular}{|l|l|l|l|l|l|l|}
\hline $\begin{array}{l}\text { Routing } \\
\text { Protocols }\end{array}$ & Classification & Objective & Summary & Simulator & $\begin{array}{l}\text { Vehicular } \\
\text { Scenario }\end{array}$ & Simulation Results \\
\hline GPCR [55] & $\begin{array}{l}\text { Source \& map } \\
\text { based protocol }\end{array}$ & $\begin{array}{l}\text { Suppressing a } \\
\text { graph } \\
\text { planarization } \\
\text { algorithm }\end{array}$ & $\begin{array}{l}\text { Greedy } \\
\text { packet } \\
\text { forwarding } \\
\text { and repair } \\
\text { strategy in } \\
\text { realistic } \\
\text { streets and } \\
\text { junctions }\end{array}$ & $\begin{array}{l}\text { ns-2 } \\
\text { simulator }\end{array}$ & $\begin{array}{l}\text { Real city } \\
\text { topology in } \\
\text { Berlin, } \\
\text { Germany }\end{array}$ & $\begin{array}{l}\text { Their algorithm } \\
\text { performs better in } \\
\text { terms of packet } \\
\text { deliver ratio and } \\
\text { average path length as } \\
\text { compared to GPSR } \\
\text { Protocol }\end{array}$ \\
\hline GeOpps & $\begin{array}{l}\text { Packet } \\
\text { buffering } \\
\text { protocol }\end{array}$ & $\begin{array}{l}\text { Addressing } \\
\text { intermittent } \\
\text { connectivity }\end{array}$ & $\begin{array}{l}\text { packet } \\
\text { forwarding } \\
\text { based on } \\
\text { vehicles' } \\
\text { movement } \\
\text { and } \\
\text { navigation } \\
\text { system }\end{array}$ & $\begin{array}{l}\text { OMNet++ } \\
\text { simulator }\end{array}$ & $\begin{array}{l}\text { Multi-agent } \\
\text { microscopic } \\
\text { traffic } \\
\text { simulator } \\
\text { (real road } \\
\text { map) }\end{array}$ & $\begin{array}{l}\text { Their algorithm } \\
\text { performs better in } \\
\text { terms of packet } \\
\text { deliver ratio, average } \\
\text { delay and average } \\
\text { path length as } \\
\text { compared to MoVe } \\
\text { and Greedy Protocols }\end{array}$ \\
\hline GeoDTN+ \\
Nav [74]
\end{tabular}




\begin{tabular}{|c|c|c|c|c|c|c|}
\hline CBF [48] & $\begin{array}{l}\text { Connectionless } \\
\text { protocol }\end{array}$ & $\begin{array}{l}\text { Reducing } \\
\text { forwarding } \\
\text { overhead }\end{array}$ & $\begin{array}{l}\text { Beaconless } \\
\text { geographical } \\
\text { forwarding }\end{array}$ & $\begin{array}{l}\text { ns-2 } \\
\text { simulator }\end{array}$ & $\begin{array}{l}\text { Highway } \\
\text { mobility } \\
\text { scenario }\end{array}$ & $\begin{array}{l}\text { Their protocol } \\
\text { forwards more packets } \\
\text { than position based } \\
\text { routing }\end{array}$ \\
\hline MoVe [51] & $\begin{array}{l}\text { Trajectory } \\
\text { based routing }\end{array}$ & $\begin{array}{l}\text { High } \\
\text { reliability and } \\
\text { low } \\
\text { dissemination } \\
\text { delay }\end{array}$ & \begin{tabular}{l|} 
Using \\
velocity \\
information \\
for \\
opportunistic \\
forwarding \\
decisions
\end{tabular} & $\begin{array}{l}\text { ns-2 } \\
\text { simulator }\end{array}$ & City scenario & $\begin{array}{l}\text { Proposed algorithm } \\
\text { performs better in } \\
\text { terms of packet } \\
\text { success rate, overhead } \\
\text { and average delay as } \\
\text { compared to the } \\
\text { existing algorithms }\end{array}$ \\
\hline DIR [68] & $\begin{array}{l}\text { Source \& map } \\
\text { based protocol }\end{array}$ & $\begin{array}{l}\text { Supporting } \\
\text { real time } \\
\text { applications }\end{array}$ & $\begin{array}{l}\text { Destination } \\
\text { discovery, } \\
\text { packet } \\
\text { forwarding } \\
\text { and route } \\
\text { maintenance }\end{array}$ & $\begin{array}{l}\text { NCTUns } \\
\text { simulator }\end{array}$ & $\begin{array}{l}\text { realistic } \\
\text { urban } \\
\text { environment }\end{array}$ & $\begin{array}{l}\text { Proposed algorithm } \\
\text { performs better in } \\
\text { terms of packet } \\
\text { delivery ratio, } \\
\text { throughput and } \\
\text { average delay as } \\
\text { compared to existing } \\
\text { algorithms }\end{array}$ \\
\hline RBVT [64] & $\begin{array}{l}\text { Source \& map } \\
\text { based protocol }\end{array}$ & $\begin{array}{l}\text { Selecting } \\
\text { high } \\
\text { connectivity } \\
\text { road segment } \\
\text { as well as } \\
\text { reducing } \\
\text { overhead in } \\
\text { dense } \\
\text { situations }\end{array}$ & $\begin{array}{l}\text { real-time } \\
\text { vehicular } \\
\text { traffic } \\
\text { information } \\
\text { to create } \\
\text { road-based } \\
\text { paths as well } \\
\text { as } \\
\text { multi-criteria } \\
\text { based } \\
\text { receiver } \\
\text { election }\end{array}$ & $\begin{array}{l}\mathrm{ns}-2 \\
\text { simulator }\end{array}$ & $\begin{array}{l}\text { Urban } \\
\text { vehicular } \\
\text { scenario }\end{array}$ & $\begin{array}{l}\text { Simulation results } \\
\text { show that the RBVT } \\
\text { performs the best } \\
\text { among existing } \\
\text { routing protocols }\end{array}$ \\
\hline SRR [75] & $\begin{array}{l}\text { Hybrid } \\
\text { Protocol }\end{array}$ & $\begin{array}{l}\text { Selecting } \\
\text { stable and } \\
\text { reliable route }\end{array}$ & $\begin{array}{l}\text { Selecting the } \\
\text { node with } \\
\text { high signal } \\
\text { strength and } \\
\text { more directed } \\
\text { to the } \\
\text { destination }\end{array}$ & $\begin{array}{l}\text { JiST/SWAN } \\
\text { simulator }\end{array}$ & $\begin{array}{l}\text { realistic } \\
\text { urban } \\
\text { environment }\end{array}$ & $\begin{array}{l}\text { The proposed } \\
\text { algorithm achieves } \\
\text { larger packet delivery } \\
\text { ratio and lower } \\
\text { latency as compared } \\
\text { to the existing } \\
\text { protocols }\end{array}$ \\
\hline CAR [58] & $\begin{array}{l}\text { Source \& map } \\
\text { based protocol }\end{array}$ & $\begin{array}{l}\text { tackling the } \\
\text { issue of } \\
\text { connectivity } \\
\text { between } \\
\text { source and } \\
\text { destination }\end{array}$ & $\begin{array}{l}\text { Route request } \\
\text { and route } \\
\text { reply are used } \\
\text { to determine } \\
\text { optimal path }\end{array}$ & $\begin{array}{l}\text { ns-2 } \\
\text { simulator }\end{array}$ & $\begin{array}{l}\text { Highway and } \\
\text { city vehicular } \\
\text { environments }\end{array}$ & $\begin{array}{l}\text { Their algorithm } \\
\text { performs better in } \\
\text { terms of average delay } \\
\text { and packet delivery } \\
\text { ratio as compared to } \\
\text { GPSR algorithm }\end{array}$ \\
\hline VADD [41] & $\begin{array}{l}\text { Packet } \\
\text { buffering } \\
\text { protocol }\end{array}$ & $\begin{array}{l}\text { selecting a } \\
\text { route with the } \\
\text { smallest } \\
\text { packet delay }\end{array}$ & $\begin{array}{l}\text { Employ the } \\
\text { concept of } \\
\text { carry-and-for } \\
\text { ward strategy } \\
\text { and the } \\
\text { predictable } \\
\text { nature of } \\
\text { vehicular } \\
\text { mobility }\end{array}$ & $\begin{array}{l}\text { ns-2 } \\
\text { simulator }\end{array}$ & $\begin{array}{l}\text { Realistic city } \\
\text { environment }\end{array}$ & $\begin{array}{l}\text { The results shows that } \\
\text { the proposed design } \\
\text { outperform existing } \\
\text { solutions in terms of } \\
\text { packet delivery ratio, } \\
\text { data packet delay and } \\
\text { protocol overhead }\end{array}$ \\
\hline
\end{tabular}




\section{Macrothink}

\section{References}

[1] ITS-Standards. Intelligent transportation systems. U.S. Department of Transportation, 1996. http://www.standards.its.dot.gov/about.asp.

[2] C.E. Perkins and E.M. Royer. Ad-hoc on-demand distance vector routing. In Proceedings of the 1999 WMCSA Workshop on Mobile Computing Systems and Application, pages 90-101.

New Orleans, LA: IEEE, 25- 26 February 1999.

http://dx.doi.org/10.1109/MCSA.1999.749281

[3] D.B. Johnson and D.A. Maltz. Dynamic source routing in ad hoc wireless networks. Mobile Computing, 353:153 - 181. 1996.

[4] T. Clausen, P. Jacquet, A. Qayyum, and L. Viennot. Optimized link state routing protocol (OLSR). IETF Internet Draft, October 2003. http://www.ietf.org/rfc/rfc3626.txt

[5] R. Morris, J. Jannotti, F. Kaashoek, J. Li, and D. Decouto. Carnet: A scalable ad hoc wireless network system. In Proceedings of the 2000 ACM SIGOPS European workshop on new challenges for the operating system, pages 65-70. Kolding: ACM, 17-20 September 2000. http://dx.doi.org/10.1145/566739.566741

[6] V.N.G.J. Soares, J.J.P.C. Rodrigues, and F. Farahmand. Performance assessment of a geographic routing protocol for vehicular delay-tolerant networks. In Proceedings of the 2012 IEEE International Conference on Wireless Communications and Networking Conference (WCNC), pages 2526-2531. France: IEEE, 12-14 December 2012. http://dx.doi.org/10.1109/WCNC.2012.6214224

[7] F. Li and Y. Wang. Routing in vehicular ad hoc networks: A survey. IEEE Vehicular Technology Magazine, 2(2):12 - 22. 2007. http://dx.doi.org/10.1109/MVT.2007.912927

[8] J. Bernsen and D. Manivannan. Unicast routing protocols for vehicular ad hoc networks: A critical comparison and classification. Pervasive and Mobile Computing, 5(1):1- 18. 2008. http://dx.doi.org/10.1016/j.pmcj.2008.09.001

[9] K.C. Lee, U. Lee, and M. Gerla. Survey of routing proto-cols in vehicular ad hoc networks. Advances in Vehicular Ad-Hoc Networks: Developments and Challenges. IGI Global, 2009.

[10] Y.H. Ho, A.H. Ho, and K.A. Hua. Routing protocols for inter-vehicular networks: A comparative study in high-mobility and large obstacles environments. Computer Communications, 31(12):2767-2780, 2008. http://dx.doi.org/10.1016/j.comcom.2007.11.001

[11] M.L. Sichitiu and M. Kihl. Inter-vehicle communication systems: A survey. IEEE Communications Surveys \& Tutorials, 10(2):88-105. 2008. http://dx.doi.org/10.1109/COMST.2008.4564481

[12] Y. Toor, P. Muhlethaler, and A. Laouiti. Vehicle ad hoc networks: Applications and related technical issues. IEEE Communications Surveys \& Tutorials, 10(3):74 - 88. 2008. http://dx.doi.org/10.1109/COMST.2008.4625806

[13] T.L. Willke, P. Tientrakool, and N.F. Maxemchuk. A survey of inter-vehicle communication protocols and their applications. IEEE Communications Surveys \& Tutorials, 11(2):3 - 20. IEEE, 2009. http://dx.doi.org/10.1109/SURV.2009.090202

[14] J. Bernsen and D. Manivannan. Routing protocols for vehicular ad hoc networks that ensure quality of service. In Proceedings of the 2008 The Fourth International Conference on Wireless and Mobile Communications, pages 1-6. Philadelphia, PA: IEEE, 01-01 October 2008. http://dx.doi.org/10.1109/ICWMC.2008.15 


\section{Macrothink}

[15] L. Jiancai, C. Feng, and X. Jiakai. The study of routing strategies in vehicular ad-hoc networks. In Proceedings of the 2010 IEEE International Conference on VWire-less Communications and Signal Processing, pages 1-5. Barcelona: IEEE, 21-23 October 2010. http://dx.doi.org/10.1109/WCSP.2010.5633461

[16] M. Azarmi, M. Sabaei, and H. Pedram. Adaptive routing protocols for vehicular ad hoc networks. In Proceedings of the 2008 IEEE International Symposium on Telecommunications, pages 825 - 830. Seoul: IEEE, 27-28 Aug 2008. http://dx.doi.org/10.1109/ISTEL.2008.4651414

[17] K. Jagadeesh, S. Siva Sathya, B. Govinda Laxmi, and B.B. Ramesh. A survey on routing protocols and its issues in vanet. International Journal of Computer Applications, 28(4):38 44. Foundation of Computer Science (FCS), 2011.

[18] Y.W. Lin, Y.S. Chen, and S.L. Lee. Routing protocols in vehicular ad hoc networks: A survey and future perspectives. Journal of Information Science and Engineering, 26(3):913 - 932. 2010.

[19] I. Lequerica, P.M. Ruiz, and V. Cabrera. Improvement of vehicular communications by using $3 \mathrm{~g}$ capabilities to disseminate control information. IEEE Network, 24(1):32-38. 2010. http://dx.doi.org/10.1109/MNET.2010.5395781

[20] J. Macker and I. Chakeres. Internet-draft, manet-iana, 2006. http://tools.ietf.org/html/draft-chakeres-manet-iana-00

[21] G. Pei, M. Gerla, and T.W. Chen. Fisheye state routing in mobile ad hoc networks. In Proceedings of the 2000 International ICDCS Workshop on Wireless Networks and Mobile Computing, pages D71-D78. Taipei: Cite-seer, 10 April 2000.

[22] C.E. Perkins and P. Bhagwat. Highly dynamic destination-sequenced distance-vector routing (DSDV) for mobile computers. ACM SIGCOMM Computer Communication Review, 24(4):234-244, 1994.

[23] S.Y. Ni, Y.C. Tseng, Y.S. Chen, and J.P. Sheu. The broadcast storm problem in a mobile ad hoc network. In Proceedings of the 1999 ACM/IEEE International Conference on Mobile Computing and Networking, pages 151-162. Seattle, WA, 15-19 1999.

[24] M. Jaap, S.and Bechler and L. Wolf. Evaluation of rout-ing protocols for vehicular ad hoc networks in city traffic scenarios. In Proceedings of the 2005 ITST International Conference on Intelligent Transportation Systems (ITS) Telecommunications, pages 1-4. Brest: ITST, 27-29 June 2005.

[25] D. Helbing, A. Hennecke, V. Shvetsov, and M. Treiber. Micro-and macro-simulation of freeway traffic. Mathematical and computer modelling, 35(5):517 - 547. Elsevier, 2002.

[26] C. Lochert, H. Hartenstein, J. Tian, H. Fussler, D. Her-mann, and M. Mauve. A routing strategy for vehicular ad hoc networks in city environments. In Proceedings of the 2003 IEEE International Symposium on Intelligent Vehicles, pages 156-161. Columbus, Ohio9-11 June 2003. http://dx.doi.org/10.1109/IVS.2003.1212901

[27] G.G. Finn. Routing and addressing problems in large metropolitan-scale internetworks. technical report isi/rr-87-i80. 1987.

[28] S. Basagni, I. Chlamtac, V.R. Syrotiuk, and B.A. Wood-ward. A distance routing effect algorithm for mobility (dream). In Proceedings of the 1998 ACM/IEEE Interna-tional Conference on Mobile Computing and Networking, pages 76-84. Dallas, TX: ACM, 25 - 30 


\section{Mll Macrothink}

October 1998. http://dx.doi.org/10.1145/288235.288254

[29] S. Bhattacharjee, K.L. Calvert, and E.W. Zegura. Self-organizing wide-area network caches. In Proceedings of the 1998 IEEE Conference on Computer and Communications, pages 600-608. San Francisco: IEEE, 29 March-2 April 1998. http://dx.doi.org/10.1109/INFCOM.1998.665080

[30] B. Blum, T. He, S. Son, and J. Stankovic. Igf: A state-free robust communication protocol for wireless sensor networks. technical report cs-2003-11, department of computer science, university of virginia. 2003.

[31] B. Jarupan and E. Ekici. Prompt: A cross-layer position-based communication protocol for delay-aware vehicular access networks. Ad Hoc Networks, 8(5):489 - 505, 2010. http://dx.doi.org/10.1016/j.adhoc.2009.12.006

[32] I. Lequerica, M. Garcia Longaron, and P.M. Ruiz. Drive and share: Efficient provisioning of social networks in vehicular scenarios. IEEE Communications Magazine, 48(11):90 - 97. IEEE, 2010. http://dx.doi.org/10.1109/MCOM.2010.5621973

[33] D. Reichardt, M. Miglietta, L. Moretti, P. Morsink, and W. Schulz. Cartalk 2000: Safe and comfortable driving based upon inter-vehicle communication. In Proceedings of the 2003 IEEE International Symposium on Intelligent Vehicle, pages 545-550. Columbus, Ohio: IEEE, 9-11 June 2003. http://dx.doi.org/10.1109/IVS.2002.1188007

[34] J. Li, J. Jannotti, D.S.J. De Couto, D.R. Karger, and R. Morris. A scalable location service for geographic ad hoc routing. In Proceedings of the 2000 International Conference on Mobile Computing and Networking, pages 120-130. Boston, Massachusetts, 6-11 August 2000.

[35] M. Kasemann, H. Fußler, H. Hartenstein, and M. Mauve. A reactive location service for mobile ad hoc networks. department of computer science, university of mannheim, tech. rep. tr-02-014. citeseer. 2002.

[36] M. Gerla, B. Zhou, Y. Z. Lee, F. Soldo, U. Lee, and G. Marfia. Vehicular grid communications: The role of the internet infrastructure. In Proceedings of the 2006 ACM International Workshop on Wireless Internet, pages 19-29. Boston, Massachusetts, August 2006.

[37] S. Jain, K. Fall, and R. Patra. Routing in a delay tolerant network. In Proceedings of the 2004 ACM Conference on Applications, Technologies, Architectures, and Protocols for Computer Communications, pages 145-158. Portland, OR, 30 August-03 September 2004.

[38] Q. Xu, T. Mak, J. Ko, and R. Sengupta. Vehicle-to-vehicle safety messaging in dsrc. In Proceedings of the 2004 ACM International Workshop on Vehicular Ad hoc Networks, pages 28-38. Philadelphia, PA, 01-01 October 2004.

[39] X. Yang, J. Liu, F. Zhao, and N.H. Vaidya. A vehicle-to-vehicle communication protocol for cooperative collision warning. In Proceedings of the 2004 IEEE International Conference on Mobile and Ubiquitous Systems: Networking and Services, pages 114 - 123. Boston, massachusetts, 22 - 26 August 2004. http://dx.doi.org/10.1109/MOBIQ.2004.1331717

[40] V.N.G.J. Soares, J.J.P.C. Rodrigues, and F. Farahmand. Geospray: A geographic routing protocol for vehicular delay-tolerant networks. Information Fusion, pages 61 - 70. Elsevier, DOI: 10.1016/j.inffus.2011.11.003.

[41] J. Zhao and G. Cao. Vadd: Vehicle-assisted data delivery in vehicular. IEEE Transactions on 


\section{Mll Macrothink}

Vehicular Technology, 57(3):1910-1922, 2008.

[42] K.Z. Ghafoor and K.A. Bakar. Inter-vehicle communication protocols for multimedia transmission. In Proceedings of the 2010 IAENG International MultiConference of Engineers and Computer Scientists, pages 1234-1239. Hong Kong: IAENG, 17-19 March 2010.

[43] I. Leontiadis and C. Mascolo. Geopps: Geographical opportunistic routing for vehicular networks. In Proceedings of the 2007 IEEE International Symposium on World of Wireless, Mobile and Multimedia Networks, pages 1- 6. Newport Beach, CA: IEEE, 23-26 June 2007. http://dx.doi.org/10.1109/WOWMOM.2007.4351688

[44] V. Cabrera, F.J. Ros, and P.M. Ruiz. Simulation-based study of common issues in vanet routing protocols. In Proceedings of the 2009 IEEE Vehicular Technology Conference, pages 1 - 5, 26 - 29 April 2007. http://dx.doi.org/10.1109/VETECS.2009.5073851

[45] J. Sanchez, P. Ruiz, and R. Marin-Perez. Beacon-less geographic routing made practical: challenges, design guidelines, and protocols. IEEE Communications Magazine, 47(8):85 91. IEEE, 2010. http://dx.doi.org/10.1109/MCOM.2009.5181897

[46] J.A. Sanchez, R. Marin-Perez, and P.M. Ruiz. Beacon-less geographic routing in real wireless sensor networks. Journal of Computer Science and Technology, 23(3):438 - 450, 2008. http://dx.doi.org/10.1007/s11276-012-0419-2

[47] V.N.G.J. Soares, J.J.P.C. Rodrigues, J.A. Dias, and J.N. Isento. Priority-based receiver-side relay election in wire-less ad hoc sensor networks. In Proceeding of the 2012 20th International Conference on Software, Telecommunications and Computer Networks (SoftCOM), pages 1-5. Croatia: IEEE, 11-13 Sept. 2012.

[48] H. Fußler, H. Hartenstein, J. Widmer, M. Mauve, and W. Effelsberg. Contention-based forwarding for street scenarios. In Proceedings of the 2004 WIT International Workshop in Intelligent Transportation, pages 15-21. Hamburg: Citeseer, 23-24 March 2004.

[49] M. Chawla, N. Goel, K. Kalaichelvan, A. Nayak, and I. Stojmenovic. Beaconless position based routing with guaranteed delivery for wireless ad-hoc and sensor networks. Ad-Hoc Networking, 212:61 - 70. 2006.

[50] D. Niculescu and B. Nath. Trajectory based forwarding and its applications. In Proceedings of the 2003 ACM International Conference on Mobile Computing and Networking, pages 260-272. San Diego, CA, 14-19 September 2003.

[51] J. LeBrun, C.N. Chuah, D. Ghosal, and M. Zhang. Knowledge-based opportunistic forwarding in vehicular wireless ad hoc networks. In Proceedings of the 2005 IEEE Vehicular Technology Conference, pages 2289-2293. Sweden: IEEE, 30 May-1 June 2005. http://dx.doi.org/10.1109/VETECS.2005.1543743

[52] B. Karp and HT. Kung. Gpsr: Greedy perimeter stateless routing for wireless networks. In Proceedings of the 2000 ACM International Conference on Mobile Computing and Networking, pages 243-254. Boston, MA, 06- 11 August 2000.

http://dx.doi.org/10.1145/345910.345953

[53] S. Olariu and M.C. Weigle. Vehicular Networks: From Theory to Practice. 1st. ed. Chapman and Hall/CRC Computer and Information Science Series, 2009.

[54] H. Fußler. Position-based Packet Forwarding for Vehicular Ad-Hoc Networks. PhD thesis, University of Mannheim, Citeseer, 2007.

[55] C. Lochert, M. Mauve, H. Fußler, and H. Hartenstein. Geographic routing in city scenarios. 


\section{Mll Macrothink}

Mobile Computing and Communications Review, 9(1):69-72. ACM, 2005. http://dx.doi.org/10.1145/1055959.1055970

[56] K.C. Lee, J. Harri, U. Lee, and M. Gerla. Enhanced perimeter routing for geographic forwarding protocols in urban vehicular scenarios. In Proceedings of the 2007 IEEE Globecom Workshops, pages 1-10. New Orleans, LA.:IEEE, 26-30 November 2007.

[57] H. Moustafa and Y. Zhang. Vehicular Networks: Techniques, Standards and Applications. 1st. ed. Auerbach Pub., 2009.

[58] V. Naumov and T.R. Gross. Connectivity-aware routing (car) in vehicular ad-hoc networks. In Proceedings of the 2007 IEEE International Conference on Computer Communications, pages $1919 \quad-\quad$ 1927. Anchorage, 6 - 12 May 2007. http://dx.doi.org/10.1109/INFCOM.2007.223

[59] Y. Ding, C. Wang, and L. Xiao. A static-node assisted adaptive routing protocol in vehicular networks. In Proceedings of the 2007 ACM international workshop on Vehicular ad hoc networks, pages 59-68. ACM, 09-14 September 2007.

[60] B.C. Seet, G. Liu, B.S. Lee, C.H. Foh, K.J. Wong, and K.K. Lee. A-star: A mobile ad hoc routing strategy for metropolis vehicular communications. In Proceedings of the 2004 LNCS International Conference on Networking Technologies, Services, and Protocols; Performance of Computer and Communication Networks; Mobile and Wireless Communications, pages 989 - 999. Athens, 9 - 14 May 2004.

[61] K. C. Lee, P. C. Cheng, J. T. Weng, L. C. Tung, and M. Gerla. Vclcr: a practical geographic routing protocol in urban scenarios. ucla computer science department, tech. rep. tr080009. 2008.

[62] K.C. Lee, M. Le, J. Harri, and M. Gerla. Louvre: Land-mark overlays for urban vehicular routing environments. In Proceedings of the 2008 IEEE Vehicular Technology Conference, pages 1-5. Calgary, Alberta, 21-24 September 2008.

[63] J. Harri, F. Filali, C. Bonnet, and M. Fiore. Vanetmo-bisim: Generating realistic mobility patterns for vanets. In Proceedings of the 2006 International Workshop on Vehicular Ad hoc Networks, pages 96-97. Los Angeles, CA, 24-29 September 2006.

[64] J. Nzouonta, N. Rajgure, G. Wang, and C. Borcea. Vanet routing on city roads using real-time vehicular traffic information. IEEE Transactions on Vehicular Technology, 58(7):3609 3626. IEEE, 2009. http://dx.doi.org/10.1109/TVT.2009.2014455

[65] H. Fußler, J. Widmer, M. Kasemann, M. Mauve, and H. Hartenstein. Contention-based forwarding for mobile ad hoc networks. Ad Hoc Networks, 1(4):351 - 369. Elsevier, 2003.

[66] M. Jerbi, S.M. Senouci, T. Rasheed, and Y. Ghamri-Doudane. Towards efficient geographic routing in urban vehicular networks. IEEE Transactions on Vehicular Technology, 58(9):5048 - 5059, 2009. http://dx.doi.org/10.1109/TVT.2009.2024341

[67] Y.B. Ko and N.H. Vaidya. Location-aided routing (lar) in mobile ad hoc networks. Wireless Networks, 6(4):307-321, 2000.

[68] Y.S. Chen, Y.W. Lin, and C.Y. Pan. Dir: Diagonal-intersection-based routing protocol for vehicular ad hoc networks. Telecommunication Systems, 10(1007):1 - 18. Springer Netherlands, 2010.

[69] K.C. Lee, U. Lee, and M. Gerla. To-go: Topology-assist geo-opportunistic routing in urban vehicular grids. In Proceedings of the 2009 IEEE International Conference on Wireless 
On-Demand Network Systems and Services, pages 11-18. Snowbird, Utah, 2-4 February 2009.

[70] C.J. Huang, I.F. Chen, K.W. Hu, H.Y. Shen, Y.J. Chen, and D.X. Yang. A load balancing and congestion-avoidance routing mechanism for teal-time traffic over vehicular networks. Universal Computer Science, 15(13):2506-2527. Universal Computer Science, 2009.

[71] X. Wang, Y. Yang, and J. An. Multi-metric routing decisions in vanet. In Proceedings of the 2009 IEEE International Conference on Dependable, Autonomic and Secure Computing, pages 551-556. Chengdu: IEEE, 12- $14 \quad$ December 2009. http://dx.doi.org/10.1109/DASC.2009.37

[72] K.Z. Ghafoor, K.A. Bakar, H.N. AL-Hashimi, and K.C. Lee. A novel delay-and reliability-aware inter-vehicle routing protocol. Network Protocols and Algorithms, 2(2):66 88, 2010.

[73] R. H. Khokhar, Md Noor Rafidah, K. Z. Ghafoor, C.-H. Ke, and M. A. Ngadi. Fuzzy assisted social-based routing for urban vehicular environments. EURASIP Journal on Wireless Communications and Networking, 178(1):100 - 118, 2011.

[74] P.C. Cheng, K.C. Lee, M. Gerla, and J. Harri. Geodtn+ nav: Geographic dtn routing with navigator prediction for urban vehicular environments. Mobile Networks and Applications, 15(1):61 - 82. Kluwer Academic Publishers, 2010.

[75] K.Z. Ghafoor, K.A. Bakar, S. Salleh, K.C. Lee, M. Mo-hamad, M. Kamat, and M. Arshad. Fuzzy logic-assisted geographical routing over vehicular ad hoc networks. International Journal of Innovative Computing, Information and Control, 8(6):1 - 26. TOKAI University, 2012.

[76] V. Naumov, R. Baumann, and T. Gross. An evaluation of inter-vehicle ad hoc networks based on realistic vehicular traces. In Proceedings of the 7th ACM International Symposium on Mobile Ad Hoc Networking and Computing, pages 108 - 119. Florence, Italy: ACM, 23-26 June 2006. http://dx.doi.org/10.1145/1132905.1132918

[77] T. Camp, J. Boleng, and V. Davies. A survey of mobility models for ad hoc network research. Wireless Communications and Mobile Computing, 2(5):483 - 502, 2007. http://dx.doi.org/10.1002/wcm.72

[78] C. Bettstetter. Mobility modeling in wireless networks: Categorization, smooth movement, and border effects. ACM SIGMOBILE Mobile Computing and Communications Review, 5(3):55-66, 2001. http://dx.doi.org/10.1145/584051.584056

[79] M. Fiore and J. H"arri. The networking shape of vehicular mobility. In Proceedings of the 9th ACM international symposium on Mobile ad hoc networking and computing (2008), pages 261-272. Hong Kong SAR, China, 27- 30 May 2008.

[80] R. Barr. An efficient, unifying approach to simulation using virtual machines. $\mathrm{PhD}$ thesis, Cornell University, Citeseer, 2004.

[81] D.R. Choffnes and F.E. Bustamante. An integrated mobility and traffic model for vehicular wireless networks. In Proceedings of the 2005 ACM international workshop on Vehicular ad hoc networks, pages 69-78. Cologne: ACM, 02-02 September 2005.

[82] T.S. Rappaport. Wireless Communications: principles and practice. 2nd. ed. Prentice Hall PTR New Jersey. 1996, 1996.

[83] K.C. Lee, U. Lee, and M. Gerla. Geo-opportunistic routing for vehicular networks. IEEE 


\section{Ml Macrothink

Communication Magazine, 10:164 - 170. IEEE, 2010.

[84] K.C. Lee, P.C. Cheng, and M. Gerla. Geocross: A geographic routing protocol in the presence of loops in urban scenarios. Ad Hoc Networks, 8(5):474 - 488. Elsevier, 2010.

[85] B. Jarupan and E. Ekici. Location and delay-aware cross-layer communication in v2i multihop vehicular networks. IEEE Communications Magazine, 47(11):112-118, 2009. http://dx.doi.org/10.1109/MCOM.2009.5307474.

\section{Copyright Disclaimer}

Copyright reserved by the author(s).

This article is an open-access article distributed under the terms and conditions of the Creative Commons Attribution license (http://creativecommons.org/licenses/by/3.0/). 